\title{
Optical Injection-Locking of VCSELs
}

\author{
Ahmad Hayat, Alexandre Bacou, \\ Angélique Rissons and Jean-Claude Mollier \\ Institut Supérieur de l'Aéronautique et de l'Espace (ISAE), \\ Toulouse \\ France
}

\section{Introduction}

Since the telecommunication revolution in the early 90s, that saw massive deployment of optical fibre for high bit rate communications, coherent optical sources have made tremendous technological advances. The technological improvement has been multi dimensional; component sizes have been reduced, conversion efficiencies increased, power consumptions decreased and integrability into compact optoelectronic sub-modules improved. Semiconductor lasers, emitting in the 1.1-1.6 $\mu \mathrm{m}$ range, have been the most prominent beneficiaries of these technological advances. This progress is a result of research efforts that consistently came up with innovative solutions and components, to meet the market demand. This in-phase, demand and supply, problem and solution and consumer need and innovation cycle, has ushered us in to the present information technology era, where stable high speed data links make the backbone of almost every aspect of life, from economy to entertainment and from health sector to defence production.

By the start of twenty-first century, a new, low cost, low power consumption and miniaturized generation of lasers had started to capture its own market share. These lasers, named Vertical-Cavity Surface-Emitting Lasers (VCSELs) due to the presence of an optical cavity which is normal to the fabrication plane, have established themselves as premier optical sources in short-haul communications such as Gigabit Ethernet, in optical computing architectures and in optical sensors. While shorter wavelength VCSEL $(<1 \mu \mathrm{m})$ fabrication technology was readily mastered, due to the ease in manipulation of AlGaAs-based materials, long wavelength VCSELs especially VCSELs emitting in the 1.3-1.5 $\mu$ range have encountered several technical challenges. There importance as low-cost coherent optical sources for the telecommunication systems is primordial, since they are compatible with the existing infrastructure.

VCSEL utilization in low-cost systems imply the application of direct modulation for high bit rate data transmission which engenders the problems of frequency chirping which increases laser linewidth and severely limits the system performance. Furthermore, relatively lower VCSEL intrinsic cut-off frequencies translated in to impossibility of achieving high bit rates. Optical injection-locking is proposed as a solution to these problems. It enhances the intrinsic component bandwidth and reduces frequency chirp considerably. 


\section{Emergence of Vertical-Cavity Lasers}

\subsection{Historical background and motivation}

It must be noted that the Vertical-Cavity Surface-Emitting Lasers (VCSELs) or simply SELs (Surface-Emitting Lasers, as they were referred to as at that time) were not proposed to overcome the bottlenecks that had hindered the progress of FTTX systems. The lasers usually used for long-haul telecommunications have cleaved structures with edge emission. Consequently they are referred to as Edge Emitting Lasers (EELs). This structure does pose some problems, e.g. the initial probe testing of these devices is impossible before there separation into individual chips. Their monolithic integration is also limited due to finite cavity length. The cavity length implies generation of undesirable longitudinal modes and the non-monolithic fabrication process implies the impossibility of fabricating laser arrays and matrices. It was specifically in order to overcome these problems that, K. Iga, a professor at that time at Tokyo University, proposed a vertical-cavity laser in 1977.

These surface-emitting lasers provided following advantages:

- Probe-testing during the manufacturing process.

- Fabrication of a large number of devices by fully monolithic processes yielding a very low-cost chip-production.

- Very small cavity length guaranteeing longitudinal single mode operation.

- Possibility of production as arrays and matrices.

- Very low threshold currents due to ultra small cavity volume.

- Monolithic integration compatibility with other devices.

- Circular far-field pattern as compared to elliptical pattern for EELs.

A pulsed operation at 77K with a threshold current of $900 \mathrm{~mA}$ was demonstrated in 1979 with a GaInAsP-InP vertical-cavity laser emitting at 1.3 $\mu \mathrm{m}$ (Soda et al., 1979). However, more pressing issues regarding the delivery of higher bit rates using the conventional EELs meant that the research into vertical-cavity lasers progressed very slowly. Consequently VCSEL research and development stagnated through out the decade that followed its first demonstration.

Continuous Wave (CW) operation of a VCSEL was presented in 1989, by Jewell et. al, for a device emitting at 850nm (Jewell et al., 1991). This VCSEL presented two unique features as compared to the previous generation of components. It had a QW-based active region and the semiconductor DBR mirrors were grown by means of Molecular Beam Epitaxy (MBE) which replaced the dielectric mirrors previously being used. The VCSEL technology then progressed steadily over the next ten years. A $2 \mathrm{~mA}$ threshold quantum-well device was presented in 1989 (Lee et al., 1989). In 1993 Continuous Wave (CW) operation for a VCSEL emitting at $1.3 \mu \mathrm{m}$ was demonstrated (Baba et al., 1993). A high power VCSEL emitting at 960nm and with an output of 20mW CW output was reported in 1996 (Grabherr et al., 1996). Despite these advances and maturity in fabrication technology, the VCSELs could not replace the EELs as optical sources for long-haul telecommunications and were hence confined to other applications such as optical computing, sensors, barcode scanners and data storage etc.

The reason for this shortcoming lies in the VCSEL physical structure that gives priority to:

- Monolithic integration favouring vertical emission

- Low threshold current

- On chip testing 
These priorities impose a set of design guidelines for VCSEL fabrication which, when implemented, induce certain unwanted and unforeseen traits in the device behaviour. These undesirable characteristics rendered the VCSEL unsuitable for utilization in prevalent telecommunication systems.

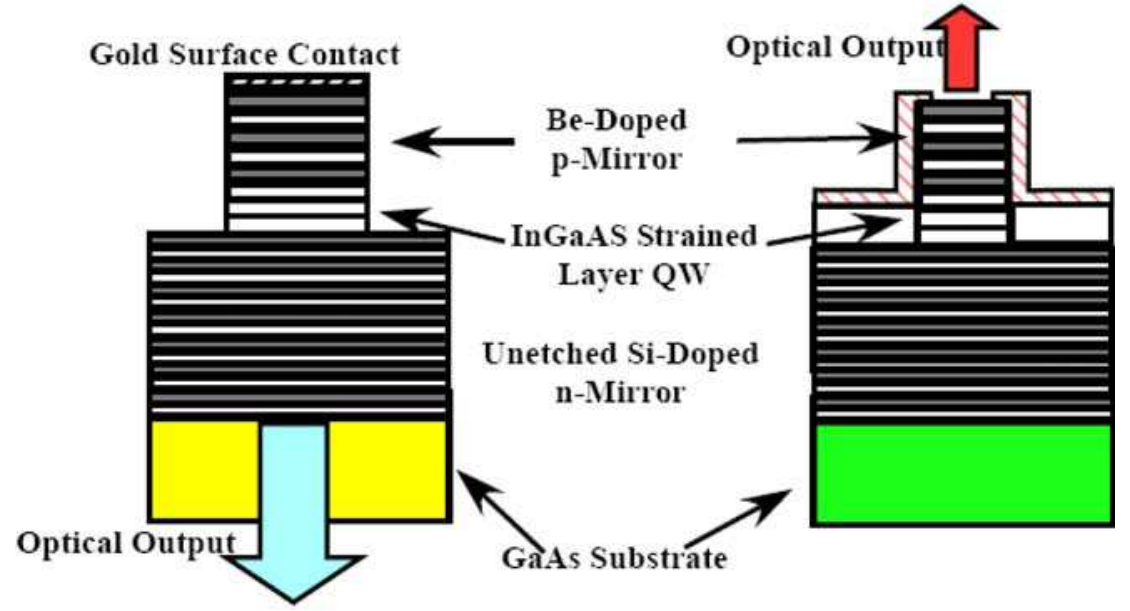

Fig. 1. An early design schematic for top-emitting and bottom-emitting VCSELs presented by Jewell et. al. in 1989.

Following is a concise analysis of these shortcomings. We would present the basic VCSEL structure that would try to achieve the above given objectives. Following this discussion we would present the drawbacks in the device performance related to the realization of design objectives. Certain remedies and improvements would then be presented in order to render the device more performing and efficient.

\subsection{VCSEL structure}

A VCSEL is essentially a gain medium based active region vertically stacked between two Distributed Bragg Reflectors (DBRs). In order to achieve a single mode operation it is proposed that the length of the active region be very small: Effectively of the order of the desired lasing wavelength. A short cavity eliminates the generation of longitudinal modes associated to Fabry-Pérot cavities. This however imposes a severe restriction on VCSEL DBR design.

The threshold gains for the surface-emitting and edge-emitting devices must be comparable regardless of the cavity length. The threshold gain of an EEL is approximately $100 \mathrm{~cm}^{-1}$. For a VCSEL of active layer thickness of $0.1 \mu \mathrm{m}$, this value corresponds to a single-pass gain of about $1 \%$. Thus for a VCSEL to lase with a threshold current density comparable to that of an EEL, the mirror reflectivities must be greater than $99 \%$ in order to ensure that the available gain exceeds the cavity losses during a single-pass.

Achieving a reflectivity of $99 \%$ with DBRs is a formidable task and thus central to the conception of low threshold VCSELs is the capacity to fabricate high reflectivity mirrors. Let's consider the example of a VCSEL operating at $850 \mathrm{~nm}$. The active region would consist of several ultra thin layers composed alternately of GaAs and AlGaAs materials. The 
difference between the refractive index of layers of a pair determines the number of pairs required to achieve a reflectivity of $99 \%$ or more. In the case of $\mathrm{AlAs}_{\mathrm{A}}-\mathrm{Al}_{0.1} \mathrm{Ga}_{0.9} \mathrm{As}$ the refractive index difference between two alternate layers is 0.6 as is shown in fig. 2 (Adachi, 1985). Consequently only 12 pairs are needed to achieve a reflectivity of $99 \%$ or more. As far as $\mathrm{AlAs}$ and $\mathrm{Al}_{\mathrm{x}} \mathrm{Ga}_{1-\mathrm{x}} \mathrm{As}$ alloys go, the situation is conducive, even desirable, for the fabrication of VCSELs using these materials. The band gap energy of $\mathrm{AlAs}^{-} \mathrm{Al}_{\mathrm{x}} \mathrm{Ga}_{1-\mathrm{x}} \mathrm{As}$ alloys is about $1.5 \mathrm{eV}$ which eventually corresponds to a wavelength in the $800-900 \mathrm{~nm}$ region.

Fabrication technology for VCSELs emitting in this wavelength band therefore has perfectly been mastered since monolithic growth of 12-15 DBR pairs does not pose serious fabrication challenges. Furthermore AlAs-GaAs alloy DBRs have an excellent thermal conductivity which allows the dissipation of heat fairly rapidly and avoids device heating which eventually could have been responsible for VCSEL underperformance.

\subsection{Performance drawbacks}

As far as the fabrication of near infrared VCSELs was concerned, the existing technologies and fabrication processes proved to be quite adequate. However, applying a similar methodology to telecommunication wavelength VCSELs proved to be much more challenging. Long wavelength VCSELs operating in the $1.1 \mu \mathrm{m}-1.6 \mu \mathrm{m}$ range are of considerable interest for optical fibre telecommunications since the hydroxyl absorption and pulse dispersion nulls for silicon optical fibres are found at $1.5 \mu \mathrm{m}$ and $1.3 \mu \mathrm{m}$ respectively. Although several material systems were considered, the combination InGaAsP-InP turned out to be the most suitable in view of the near perfect lattice match. The active layer is composed of the $\mathrm{In}_{1-\mathrm{x}} \mathrm{Ga}_{\mathrm{x}} \mathrm{As}_{\mathrm{y}} \mathrm{P}_{1-\mathrm{y}}$ quaternary alloy. By varying mole fractions $\mathrm{x}$ and $\mathrm{y}$, almost any wavelength within the 1.1-1.6 $\mu \mathrm{m}$ can be selected.

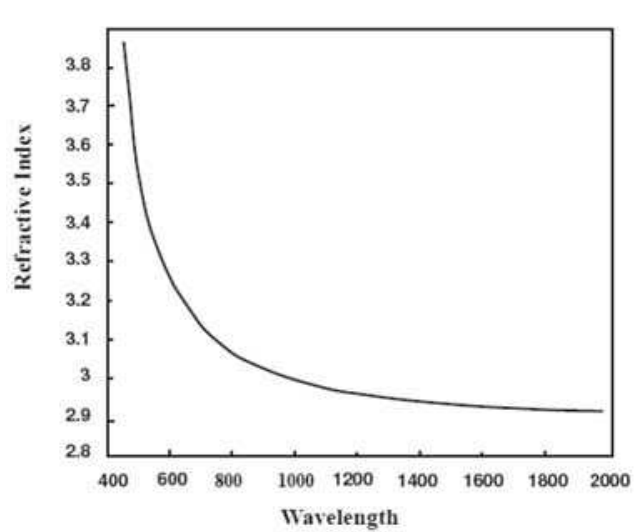

(a) Refractive Index of AlAs

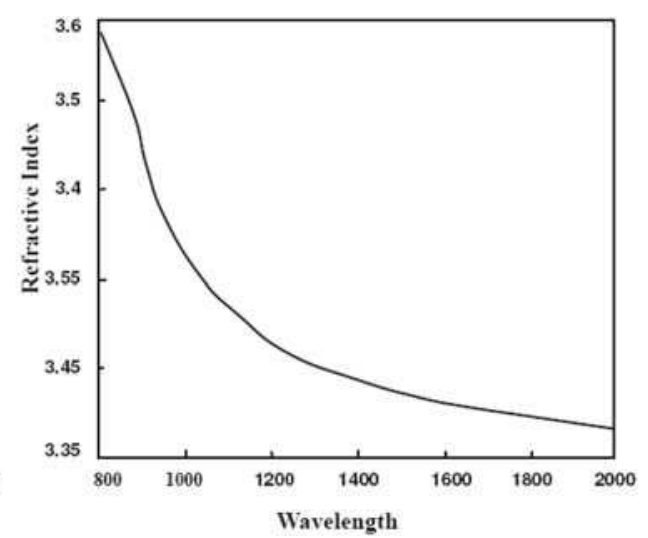

(b) Refractive Index of $A l_{0.1} G a_{0.9} A s$

Fig. 2. Refractive indices of $\mathrm{AlAs}$ and $\mathrm{Al}_{0.1} \mathrm{Ga}_{0.9} \mathrm{As}$ as a function operating wavelengths.

\subsection{DBR growth}

Only 12-15 $\mathrm{AlAs}-\mathrm{Al}_{x} \mathrm{Ga}_{1-x} \mathrm{As}$ pairs are needed to fabricate a DBR with a $99 \%$ reflectivity. By contrast, the refractive index difference between an InP- InGaAsP pair is only 0.3 and hence more than 40 pairs would be needed to achieve a reflectivity of $99 \%$. The problem 
consequently encountered concerns thermal properties of InP-based materials that intervene to affect the process in following ways (Shau et al., 2004), (Piprek, 2003):

- For the fabrication of long wavelength VCSELs, there are mainly $\operatorname{In}_{1-x} \mathrm{Ga}_{x} \mathrm{As}_{\mathrm{y}} \mathrm{P}_{1-\mathrm{y}}$ alloys available which have to be grown on InP substrates. Due to the effects of non negligible Auger's recombination effects and intra-valence band absorption, these materials suffer from temperature-dependent losses.

- The thermal conductivity is greatly reduced due to alloy disorders which causes phonon scattering. This reduction in thermal conductivity is particularly adverse for effective heat sinking through the VCSELs' DBRs usually having a thickness of several $\mu \mathrm{ms}$.

- AlAs- $\mathrm{Al}_{x} \mathrm{Ga}_{1-x} \mathrm{As}$ DBRs have a good thermal conductivity and could be thinner but due to lattice mismatch could not be grown on the InP substrate.

DBR growth has been one of the fundamental problems regarding the fabrication of long wavelength VCSELs that has hampered the entry of VCSELs in high-speed data, command and telecommunications domain.

\subsection{Optical and electrical confinement}

Growing stacks of DBRs was not the only problem encountered by VCSEL manufacturers. One of the primary objectives of VCSEL design was to fabricate short cavity single mode devices. The short cavity did eliminate the undesirable longitudinal modes but it gave birth to another unforeseen problem. Initial VCSEL designs suggested that the carriers and the photons share a common path traversing the DBRs. This led to the heating of certain zones of the DBRs due to carrier flow and resulted in a variable refractive index distribution inside the VCSEL optical cavity. This phenomenon is known as "Thermal Lensing". Instead of being concentrated in the centre in the form of a single transverse mode, the optical energy is repartitioned azimuthally inside the optical cavity. This particular optical energy distribution is observed in the form of transverse modes. Higher bias currents therefore imply high optical power and in consequence a higher number of transverse modes.

An oxide-aperture is employed, principally in shorter wavelength emission VCSELs, in order to block the unwanted transverse modes. The oxide-aperture diameter then determines the multimode or single mode character of a VCSEL. VCSELs having oxide aperture diameter greater than $5 \mu \mathrm{m}$ exhibit multimode behaviour. It can also be inferred from the above discussion that for the type of VCSELs employing the oxide-aperture technology for optical confinement, single mode VCSELs almost always have emission powers less than those of multimode VCSELs.

The problems of optical and electrical confinement are hence interrelated. It is evident that in order to attain single mode emission the thermal lens effect must be avoided. This can only be achieved by segregating the carrier and photon paths. Although challenging technically, it can be achieved using a tunnel junction. The concept and functioning of a tunnel junction is explained in the following sub-section.

\subsection{The tunnel junction}

The "Tunnel Junction" was discovered by L. Esaki in 1951 (Esaki, 1974) and the tunnel junction diodes used to be labeled "Esaki Diodes" for quite some time after this discovery (Batdorf et al., 1960), (Burrus, 1962). Esaki observed the tunnel junction functioning while working on Ge layers but soon after his discovery, tunnel junction diodes were presented by 
other researchers on other semiconductor materials such as GaAs, InSb, Si and InP. The tunnel junction is formed by joining two highly doped (degenerate) " $p$ " and " $n$ " layers. It has a particular current-voltage characteristic curve. A negative differential resistance region $(-\mathrm{dI} / \mathrm{dV})$ over part of the forward characteristics can be observed.

In the case of a VCSEL the tunnel junction serves a "Hole Generator". Under the tunnel effect, the electrons move from valence band (doped $\mathrm{p}^{++}$) to conduction band (doped $\mathrm{n}^{++}$), leaving holes in their place. Fig.1.12 shows the schematic diagram of a tunnel diode in reverse bias conditions. The existence of a tunnel junction in a VCSEL presents following advantages:

- It reduces the intra valence band absorption due to P doping.

- It serves to reduce the threshold current, by improving the carrier mobility.

- It is used for electrical as well as optical confinement.

Due to these properties, the tunnel junction has become an integral part of long wavelength VCSELs.

2.7 Technological breakthroughs and advances in long wavelength VCSEL fabrication Although by the start of the 21st century serial production and delivery of VCSELs was in full flow for diverse applications, they had failed to fulfil the two following essential criteria for utilization in optical networks.

- They did not emit in the $1.3 \mu \mathrm{m}$ and $1.5 \mu \mathrm{m}$ range: The so-called "Telecoms Wavelengths". This meant not only definition and standardization of new standards at $850 \mathrm{~nm}$ wavelength but also the deployment and manufacturing of a host of optical components such as optical fibres, couplers, multiplexers and photodiodes compatible with the $850 \mathrm{~nm}$ emission range.

- As has been explained above, transverse-mode operation starts to manifest itself from a few milli-amperes above the threshold current rendering the VCSELs multimode in character. This multimodality is disconcerting in two ways:

- It reduces the effective channel bandwidth hence reducing the maximum deliverable bit rate.

- It requires the utilization of multimode optical fibre which although being less expensive than the single mode fibre, affects the VCSEL operation in another way. When high optical powers are injected in a multimode fibre, several undesired fibre modes are excited thus reducing the effective bandwidth.

It is clear from the above discussion that a suitable substitute for EELs, for applications in short to medium distance optical fibre networks, must possess the following properties:

- It must emit at either $1.3 \mu \mathrm{m}$ or at $1.5 \mu \mathrm{m}$ wavelength so that the existing standards, infrastructure, optoelectronic components and devices could be utilized.

- It must have a single mode emission spectrum so as to profit from the high bandwidths offered by the employment of single mode optical fibres.

As late as 2000, there were no serial production and mass deployment of VCSELs that fulfilled these two essential criteria. As has been discussed above, this was due to the technical challenges posed by a combination of several different factors which rendered the fabrication of long wavelength VCSEL devices very difficult.

\subsection{Emergence of long wavelength VCSELs}

Regarding the manufacturing of long wavelength VCSELs, several different research groups kept trying to realize long wavelength emission devices. In 1993, Iga et al. demonstrated the 
CW operation of a 1.3 $\mathrm{mm}$ InGaAs-InP based VCSEL at 77K (Soda, 1979). The upper DBR consisted of 8.5 pairs of p-doped MgO-Si material with Au-Ni- Au layers at the top while the bottom DBR consisted of 6 pairs of n-doped SiO-Si material (Dielectric Mirror). In 1997, Salet et.al demonstrated the pulsed room-temperature operation of a single mode InGaAsInP VCSEL emitting at $1277 \mathrm{~nm}$. The bottom mirror consisted of n-doped InGaAsP-InP material grown epitaxially to form a 50 pair DBR mirror with a $99.5 \%$ reflectivity (Salet et al., 1997).

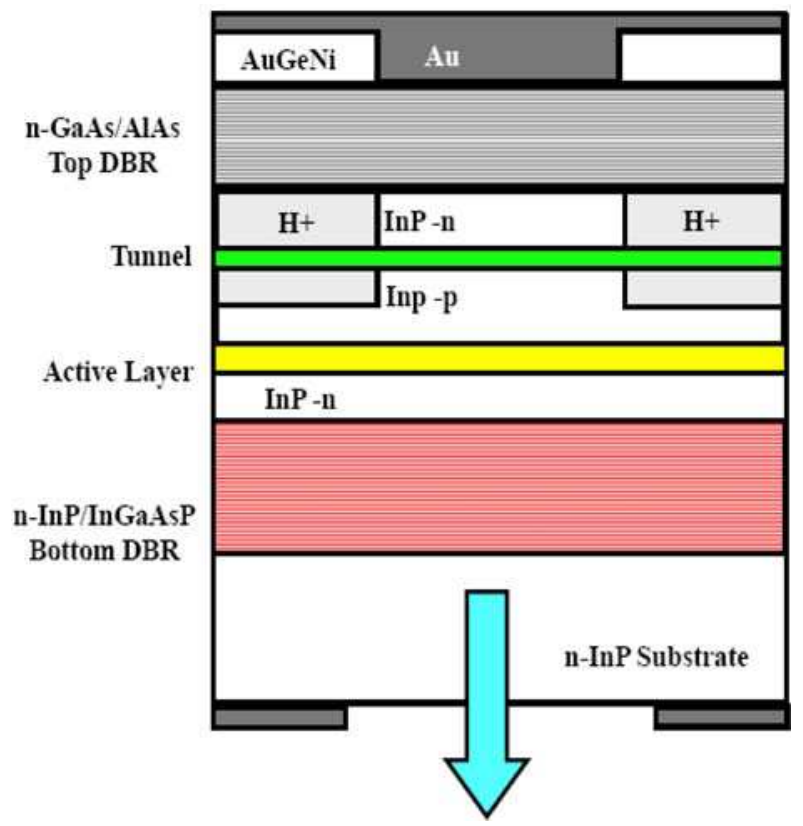

Fig. 3. A long wavelength VCSEL with a tunnel junction emitting at $1.55 \mu \mathrm{m}$ presented by Boucart et. al in 1999.

The device threshold current at $300 \mathrm{~K}$ was $500 \mathrm{~mA}$. The top mirror was realized using $\mathrm{p}$ doped $\mathrm{SiO}_{2}$-Si reflectors. A year later, in 1998, Dias et al. reported the growth of InGaAsPInP, AlGaInAs-AlInAs and AlGaAsSb-AlAsSb based DBRs on InP substrates to achieve reflectivities up to $99.5 \%$ (Dias et al., 1998). Soon afterward, in 1999, Boucart et al extended their previous work to demonstrate the room temperature $\mathrm{CW}$ operation of a $1.55 \mu \mathrm{m}$ VCSEL. In this case the top DBRs consist of 26.5 n-doped GaAs-AlAs pairs which were grown directly on an $\mathrm{n}$-InP substrate (Metamorphic mirrors). A tunnel junction was fabricated to localize the current injection. The bottom mirror consisted of 50 pairs of ndoped InGaAsP-InP layers having a reflectivity of $99.7 \%$. The device had a threshold current of only $11 \mathrm{~mA}$ and had been fabricated using gas-based Molecular Beam Epitaxy (MBE) (Boucart et al., 1999).

The tunnel junction proved benificial in two ways:

- It enabled the utilization of two n-doped DBRs;

- Once the conductive properties of the tunnel junction were neutralized using $\mathrm{H}+$ ion implantation, it served to localize the current injection without having to etch a mesa. 
The resulting device was therefore coplanar in structure. It can be ascertained from Table.1.1 that several different materials such as InGaAsP, InGaAsAl, InGaAsSb and InGaAsN were chosen to fabricate the active layer. The material choice for DBRs and the fabrication processes were equally diverse. Although most of the research groups chose "Monolithic Integration Techniques" for the fabrication of VCSELs, "Wafer Fusion", and "Fusion Bonding" were also applied.

Meanwhile, in 1998, the Institute of Electrical and Electronics Engineers (IEEE) defined the "1000BASEX-Gbps Ethernet over Fibre-Optic at 1Gbit/s" standard. This standard for the transmission of "Ethernet Frames" at a rate of at least one Gbps was defined using light sources emitting at $850 \mathrm{~nm}$. The definition of Gigabit Ethernet standards using 850nm optical sources boosted the research and development of near infrared emission VCSELs. By the year 2000, 850nm VCSELs had firmly established themselves as standard optical sources for short-haul communication applications. This development was a setback for ongoing research in long wavelength VCSELs and as a result many research groups shifted their focus from long wavelength VCSEL development to other emerging fields. Furthermore, the research focus, even in the long wavelength VCSEL development field, shifted toward a new dimension. Long wavelength VCSELs were no longer being developed solely as telecommunication sources, an emerging field of spectroscopy was beginning to play an increasingly important part in eventual long wavelength VCSEL applications.

\subsection{Vertilas VCSELS}

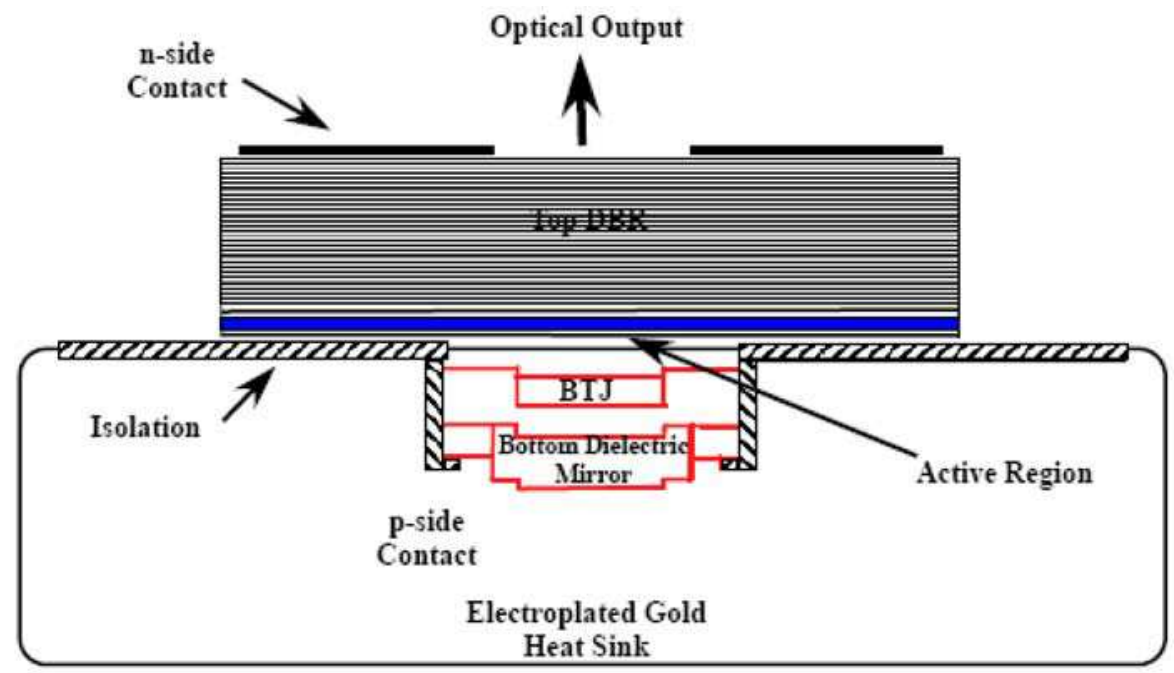

Fig. 4. A Vertilas BTJ structure with an emission wavelength of $1.55 \mu \mathrm{m}$ [28].

Although long wavelength VCSEL operation using a tunnel junction device was already demonstrated by Boucart et al. in 1999, Ortsiefer et al. presented a variation to this concept. Soon the single mode room temperature operation of an InP-based VCSEL operating at $1.5 \mu \mathrm{m}$ was demonstrated by the same research group (Ortsiefer et al., 1999), (Ortsiefer et al., 2000). The top DBR is composed of 34.5 InGaAlAs-InAlAs pairs. The bottom mirror is comprised of 2.5 pairs of CaF2-Si with Au-coating. The gold coating, apart from serving as a 
high reflectivity mirror (99.75\%), serves as an integrated heat sink (Shau et al., 2004). The successful incorporation of tunnel junction in the long wavelength VCSEL design proved to be the technical breakthrough that would present VCSELs as standard devices for short to medium distance optical fibre communications. By 2002 Vertilas was delivering $1.55 \mu \mathrm{m}$ single mode VCSELs for 10Gbps operation.

\subsection{BeamExpress VCSELS}

The manufacturing of a long wavelength VCSEL requires the growth of an InP-InGaAsP alloy active region on an InP substrate. These alloys however are difficult to grow as DBR stacks above and below the active region since the restrictions imposed by the material thermal conductivity render proper device functioning impossible. On the other hand, AlAs- $\mathrm{Al}_{x} \mathrm{Ga}_{1-x} \mathrm{As}$ DBRs have a good thermal conductivity but they can not be monolithically grown on InP-based substrates due to lattice mismatch. The solution to the matching of disparate materials to optimize VCSEL performance was developed at the University of California Santa Barbara (UCSB) in 1996 by Margalit et al. (Margalit et al., 1996). The technique utilized is known as "Wafer Fusion" or "Wafer Bonding" and consists of establishing chemical bonds directly between two materials at their hetero-interface in the absence of an intermediate layer (Black et al., 1997). The first demonstration constituted of fabrication of a $1.55 \mu \mathrm{m}$ VCSEL. The device was fabricated by wafer fusion of MOVPEgrown InGaAsP quantum well active region to two MBEgrown AlGaAs-GaAs DBR reflectors (Margalit et al., 1996).

By applying a variant of the "Wafer Fusion" technique in 2004, Kapon et. al demonstrated that it was possible to grow separate components of a VCSEL cavity on separate host substrates (Syrbu et. al, 2004), (Syrbu et. al, 2005). These separate components were then bonded (fused) together to construct the complete VCSEL optical cavity. This process was developed at the Ecole Polytechnique Fédérale de Lausanne (EPFL) and patented as "Localized Wafer Fusion". Fig. 5 presents the structure of a BeamExpress VCSEL with an emission wavelength of $1.55 \mu \mathrm{m}$. This is a double intracavity contact single-mode VCSEL with coplanar access. The InP-based optical cavity consists of five InAlGaAs quantum wells. The top and bottom DBRs comprise of 21 and 35 pairs respectively and are grown by MetalOrganic Chemical Vapor Deposition (MOCVD) epitaxy method. Using the technique of localized wafer fusion, the top and the bottom AlGaAs-GaAs DBRs are then bonded to the active cavity wafer and the tunnel junction mesa structures. Using VCSELs with double intracavity contacts has its own advantages. These contacts are much nearer to the active region than the classical contacts. Their utilization combined with the presence of tunnel junction allows having lower series resistance as compared to oxidized-aperture VCSELs. Due to this proximity of the contacts to the active region these VCSELs tend to have high quantum efficiency. Their location near the active region results in no current passage through DBRs.

The process used for the fabrication of Beam Express VCSELs is not monolithic. The bottom AlGaAs-GaAs DBR is grown on the GaAs substrate. The InP-based cavity is then bonded to this DBR. After the growth of an isolation layer on the active region, the epitaxially grown AlGaAs-GaAs top DBR is fused to complete the optical cavity. This double fusion increases the complexity of the fabrication process but it presents certain advantages. Waferfusion allows replacing the InAlGaAs DBRs by GaAs DBRs. Not only the GaAs DBRs have a better thermal conductivity, they are much cheaper than InAlGaAs DBRs which allows increasing the performance and decreasing the cost of the component at the same time. The biggest 
advantage of "Wafer Fusion" is the possibility of serial production of VCSELs which further serves to reduce the component cost.

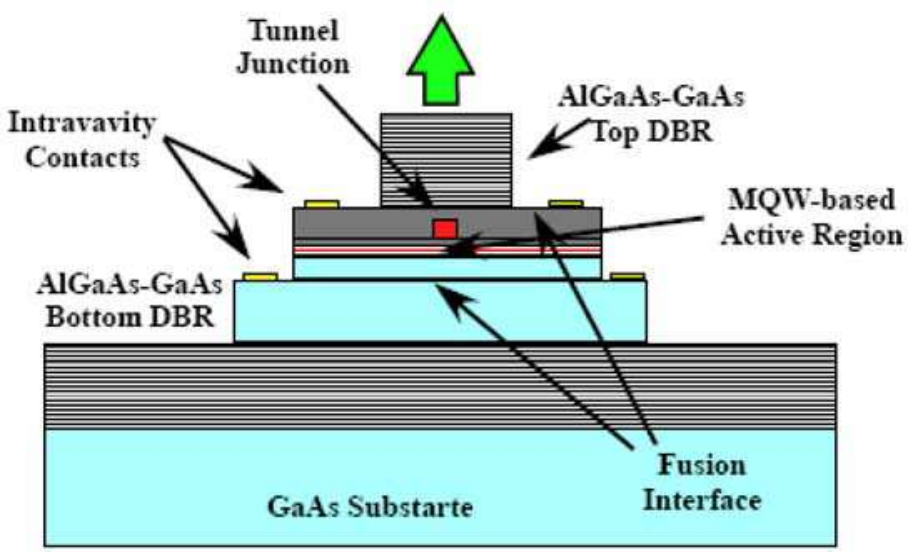

Fig. 5. Schematic diagram of a wafer-fused Beam-Express VCSEL with an emission wavelength of $1.5 \mu \mathrm{m}$.

\subsection{RayCan VCSELS}

Starting as a spin-off company from the Korean government funded Electronics and Telecommunications Research Institute (ETRI) in 2002, RayCan launched an ambitious project for manufacturing of long wavelength VCSELs. Instead of using the above described specialized technologies for long wavelength VCSEL manufacturing, RayCan decided to embark upon a different course. They decided to monolithically grow InAlGaAs DBRs and an InGaAs-based quantum well active region on an InP substrate. As has been discussed above, this technique was previously not considered because in order to achieve $99 \%$ reflectivity using InAlGaAsbased DBRs, a growth of more than 40 pairs is needed. RayCan employed Metal-Organic Chemical Vapour Deposition (MOCVD) technique to fabricate a long wavelength VCSEL.

For $1.55 \mu \mathrm{m}$ VCSELs, the top and bottom DBRs were grown as 28 and 38 pairs of un-doped InAlGaAs-InAlAs schemes. The top and bottom DBRs consisted of 33 and 50 layers respectively for $1.3 \mu \mathrm{m}$ emission VCSELs. The $0.5 \lambda$ thick active region consists of seven pairs of strain-compensated (SC) InAlGaAs quantum wells (Park et al., 2006). The lower number of top DBRs in both the VCSELs was compensated by using an InAlGaAs phasematching layer and Au metal layer. Fig. 6 presents the structure of a RayCan VCSEL emitting at

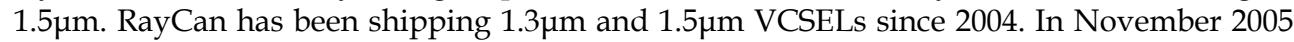
RayCan shipped its first 10GBit/s long wavelength CWDM VCSEL module.

\subsection{Long wavelength VCSEL direct modulation}

Up to this point we have discussed the prospects of long wavelength VCSELs in the context of high bit rate data delivery over medium and short distance links. It would not be an exaggeration to state that consumer demand for multimedia and interactive applications and therefore bandwidth has increased to an unprecedented level. Current electricalelectrical infrastructures can not support this demand. The major obstacle in switching from 
electrical/ hertzian systems to optical/fibred systems is the cost of the coherent optical source compatible with existing infrastructure. Recent advances in the fabrication, development and serial production of VCSELs emitting at $1.3 \mu \mathrm{m}$ and $1.5 \mu \mathrm{m}$ have paved the way for future FTTX systems.

Having been able to solve the problem at component level, by developing reliable long wavelength VCSELs, the next logical approach is the development of new systems incorporating these components. Conventionally the EELs used in the long-haul fibre links are externally modulated i.e. the photon generation process inside the cavity is independent of the modulation mechanism. While being extremely effective, this method necessitates the utilization of an external modulator which increases the system cost. Such a scheme is inherently unfeasible for FTTX systems due to the cost of the external modulators. The elimination of external modulators as a component of choice for FTTX systems decrees the employment of direct modulation techniques. In this technique the laser diode bias current is varied to achieve the optical output intensity variation. Apparently the scheme is simple and easy to implement, but when put into practice, it presents two major problems which are detailed in the following two sub-sections.

\subsection{Phase-amplitude coupling}

Semiconductor lasers, whether EELs or VCSELs, are different from other lasers in one respect. The refractive index of a semiconductor laser depends on the carrier concentration inside the cavity. The carrier concentration variation affects the refractive index of the cavity which eventually changes the emission wavelength of the component. The consequences of this uniqueness manifest themselves during the process of direct modulation. A variation in bias currents varies the optical output power as well as the optical frequency of the cavity. These variations are proportional to the variation in carrier concentration and therefore the bias current.

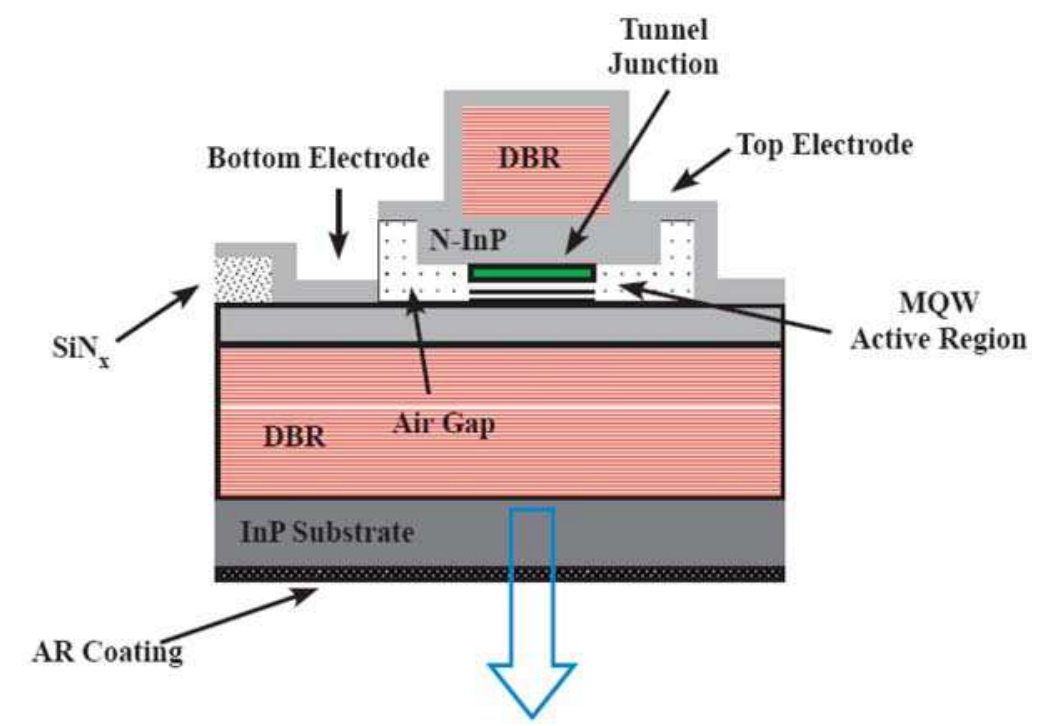

Fig. 6. MOVCD Grown monolithic structure of a 1.5 $\mu$ m RayCan VCSEL. 
The device is modulated in amplitude and frequency at the same time. This phenomenon of "Phase-Amplitude Coupling" or the dynamic shift of the lasing frequency during modulation is known as "Frequency Chirping" or simply "Chirping".

Chirping broadens the linewidth of a laser. The extent to which a pulse broadens depends upon the amplitude of the modulating signal. Larger modulation amplitudes result in linewidths of the order of $\mathrm{GHz} 1$. This spectral broadening at the time of modulation becomes more pronounced during the passage of the modulated pulse through an optical channel and the effective channel bandwidth is reduced. Direct modulation while being costeffective proves to be inefficient, in terms of deliverable bit rates, when compared to external modulation.

\subsection{Intrinsic modulation limits}

A semiconductor optical cavity, in essence, is a resonator. Like every resonator, or electrical circuit for that matter, its frequency response depends on its intrinsic parameters. In case of semiconductor lasers these parameters might be cavity volume, photon and electron populations, group velocity, gain compression factor etc. When directly modulated, a laser can not better the modulation frequency response already defined by these intrinsic parameters. On the other hand, the utilization of an external modulator provides a means to bypass the laser intrinsic parameters. The modulation response (or the deliverable bit rate) of the system is then defined by the external modulator and not the laser.

\subsection{Long wavelength VCSEL optical injection-locking}

It is clear from the description of the two above given problems that a viable optical system must minimize the effects of "Amplitude-Phase Coupling" and "Intrinsic Modulation Limits" in order to be efficient and acceptable. Once injection-locked, the master laser holds the frequency of the follower laser and makes it immune to carrier variations. This isolation from carrier variations appears as the reduction of chirp during direct modulation. In 1984, Lin et al. demonstrated the reduction of frequency chirping in a directly modulated semiconductor laser by the application of injection-locking technique (Lin et al., 1984).

Henry presented an approximate formula for the calculation of resonance frequency of optically injection-locked semiconductor lasers (Henry et al., 1985) but its significance was not appreciated at that time until Simpson and Meng demonstrated bandwidth and resonance frequency enhancements in late 90's (Simpson et al., 1996), (Meng et al., 1998). In 2002, a research group in University of California Berkley (UCB), led by Connie J. ChangHasnain reported the first optical injection-locking of a long wavelength VCSEL for 2.5Gbps transmission (Chang et al., 2002).

In 2003 long wavelength VCSEL chirp reduction and bandwidth enhancement were presented by the same research group (Chang et al., 2003) but there was a marked technical difference from their first publication. Whereas the first time optical injection-locking of a long wavelength VCSEL was carried-out using an identical VCSEL, the second demonstration used a Distributed FeedBack (DFB) laser to injection-lock a long wavelength VCSEL. The group has extensively published on the subject of the optical injection-locking of long wavelength VCSELs, but this pattern of locking a VCSEL with a DFB has remained unchanged since.

Several optical injection-locking studies regarding semiconductor lasers have reported frequency-chirp reduction (Lin et al., 1984), (Sung et al., 2004) increased RF link gain 
(Chrostowski et al. 2003), (Chrostowski et al. 2007), improved relative intensity noise (Yabre et al., 2000) and diminished non-linear distortion (Chrostowski et al. 2007). Although the utilization of a DFB laser to injection-lock a VCSEL is excellent for demonstration of phenomena related to optical injection-locking, its practical application presents two major drawbacks. Without immediately entering into the details of these drawbacks, it can be logically inferred that both these drawbacks are related to the utilization of the DFB laser.

First of all the physical symmetry of the two lasers used is not the same. The VCSELs are a vertical emission device while the DFB lasers emit in the horizontal direction. This asymmetry renders the integration of an optical injection-locking system consisting of a DFB laser and a VCSEL very difficult. The second reason, of course, is the cost. One of the reasons of employing VCSELs in optical networks for high-speed data communication is their cost-effectiveness. Utilization of a DFB laser to improve the transmission and the component characteristics compromises this very objective. Due to these reasons despite all these advances regarding this very potent combination of semiconductor lasers and optical injection-locking, the phenomenon and its practical applications have not got any commercial breakthrough as yet.

With the arrival of Vertical-Cavity Surface-Emitting Lasers (VCSELs) on the commercial scene as low-cost, integrable sources, the efforts to revive the optical injection-locking phenomena were once again undertaken and follower VCSEL resonance frequencies ranging from $27 \mathrm{Ghz}$ to $107 \mathrm{GHz}$ have been reported in recent years (Chrostowski et al. 2007). The problem of non-integrability however is still unresolved due to the utilization of a distributed feedback (DFB) laser as master optical source to injection-lock a follower VCSEL. The DFB lasers have horizontal optical cavities. This physical asymmetry renders the monolithic integration very complicated. On the other hand the utilization of a powerful DFB laser compromises the economy of the setup by increasing the cost dramatically and fails the purpose of using a VCSEL in the first place. Clearly the solution to afore-mentioned problems would be to try a VCSEL-by-VCSEL optical injection-locking approach.

\section{VCSEL rate equations}

The previous chapter introduced the overall historical background of the subject and the motivation for undertaking this research work. In this chapter we will present a complete theoretical analysis of the optical injection-locking phenomenon in semiconductor lasers. A semiconductor laser cavity is essentially a resonator and its input (electrons) and output (photons) can be demonstrated to be interrelated to each other via cavity parameters. Like any other resonator cavity, the quality factor " $Q$ " and the resonance frequency of this cavity can be controlled by manipulating its physical dimensions or intrinsic parameters.

Ordinarily, the only externally manipulable variable is the electron concentration that can be varied by changing the bias current. During the optical injection-locking process the internal parameters of the cavity are changed by varying the photon concentration inside the cavity. Since the locking effect is the result of interaction between two optical fields, the phase difference between the master and follower VCSELs can also be varied to achieve the desired effect.

Ordinarily, the only externally manipulable variable is the electron concentration that can be varied by changing the bias current. During the optical injection-locking process the internal parameters of the cavity are changed by varying the photon concentration inside the cavity. Since the locking effect is the result of interaction between two optical fields, the phase 
difference between the master and follower VCSELs can also be varied to achieve the desired effect.

$$
\begin{gathered}
\frac{d N(t)}{d t}=\frac{\eta_{i} I}{q V_{a c t}}-\left(A+B N(t)+C N(t)^{2}\right) N(t)-v_{g} G S(t) \\
\frac{d S(t)}{d t}=\Gamma \beta B N(t)^{2}+\Gamma v_{g} G S(t)-\frac{S(t)}{\tau_{P}}
\end{gathered}
$$

Where $\mathrm{N}(\mathrm{t})$ and $\mathrm{S}(\mathrm{t})$ are the electron and photon densities, $\eta_{i}$ the internal quantum efficiency, $\mathrm{q}$ the electron charge, $\mathrm{V}_{\text {act }}$ the active region volume, $\mathrm{v}_{\mathrm{g}}$ the group velocity, $\beta$ the spontaneous emission coefficient, $\Gamma$ the confinement factor and $\tau_{P}$ the photon lifetime.

The spontaneous emission rate, $R_{s p}$ is defined in terms of the constants $A, B$ and $C$ where A represents the Shockly-Read-Hall non-radiative recombination coefficient, $\mathrm{B}$ the bimolecular recombination coefficient and $C$ the Auger non-radiative recombination coefficient. The gain G can be expressed as

$$
G=a_{0} \frac{N(t)-N_{t r}}{1+\epsilon S(t)}
$$

Where $\mathrm{N}_{\text {tr }}$ is the transparency carrier density, $\mathrm{a}_{0}$ the differential gain coefficient and $\varepsilon$ the gain compression factor.

A third equation describing the phase behaviour of the device can be introduced as follows:

$$
\frac{d \phi(t)}{d t}=\frac{\alpha_{H} \Gamma v_{g} a_{0}}{2}\left(N(t)-N_{t r}\right)
$$

$\alpha_{H}$ is the "Phase-Amplitude" coupling factor and is referred to as "Henry's Factor". It might be important to note here that equation 2.4 is not a coupled equation i.e. the term does not appear in equations 2.1 and 2.2. Lang proposed the utilization of three equations, instead of two, to model an optically injection-locked system (Lang, 1982). Lang's equations coupled the electric field variations in the cavity directly to carrier and phase variations and as such rendered the physical interpretation of the phenomenon somewhat cumbersome. In 1985, P. Gallion et al. presented the optical injection-locking rate equations that replaced cavity electrical field by photon number (Gallion \& Debarge, 1985), (Gallion et al., 1985). Following the injection of optical power in the optical cavity, the dynamics of the follower laser change. This change can be mathematically presented by modifying the VCSEL rate equations to compensate for optical injection.

$$
\begin{aligned}
\frac{d N(t)}{d t} & =\frac{\eta I}{q V_{\text {act }}}-\left(A+B N(t)+C N(t)^{2}\right) N(t)-v_{g} G S(t) \\
\frac{d S(t)}{d t} & =\Gamma v_{g} G S(t)-\frac{S(t)}{\tau_{P}}+\frac{v_{g}}{L} \sqrt{S(t) S_{i n j}} \cos (\theta)+\Gamma B \beta N(t)^{2} \\
\frac{d \phi(t)}{d t} & =\frac{\alpha_{H} \Gamma v_{g} a_{0}}{2}\left(N(t)-N_{t r}\right)-\Delta \omega-\frac{v_{g}}{2 L} \sqrt{\frac{S_{\text {inj }}}{S(t)}} \sin (\theta)
\end{aligned}
$$


It must be remarked that while the equation concerning the carrier density remains unchanged, the equations regarding the phase and the photon density are modified to accommodate for the effects of external light injection.

Two very important parameters of note, $S_{\text {inj }}$ and $\theta$ are added to equations 2.6 and 2.7. $S_{\text {inj }}$ represents the photon density injected inside the follower VCSEL optical cavity while $\theta$ denotes the phase difference between the master and follower optical fields so that:

$$
\begin{gathered}
\theta=\phi_{\text {inj }}-\phi(t) \\
\Delta \omega=\omega_{\text {Master }}-\omega_{\text {Follower }}
\end{gathered}
$$

Apart from frequency detuning, phase difference and injected optical power, the fourth parameter which characterizes an optically injection-locked system is the "coupling coefficient" of a laser. It is defined as $\mathrm{k}_{\mathrm{c}}$ and can be expressed mathematically as

$$
k_{c}=\frac{v_{g}}{2 L}
$$

This coefficient describes the rate at which the injected electric field adds to the follower cavity electric field as a function of the VCSEL optical cavity length. ' $L$ ' is the length of the VCSEL optical cavity.

\subsection{Locking Range Calculations}

Solving equations (5) and (6) in the steady-state regime which renders $\frac{d S}{s T}$ and $\frac{d N}{s T}$ equal to zero gives the very important parametric equation:

$$
\Delta \omega=k_{c} \sqrt{\frac{S_{\text {inj }}}{S}}\left[\sin (\theta)-\alpha_{H} \cos (\theta)\right]
$$

The dependence of equation (11) on $\alpha_{H}$ can be elaborated by using the linear combination property for sinuses and cosines. Using this property we can write that:

$$
\Delta \omega=k_{c} \sqrt{\frac{S_{i n j}}{S}}\left[\sqrt{1+\alpha_{H}^{2}}\right] \sin \left(\theta-\tan ^{-1} \alpha_{H}\right)
$$

This relation is important because it helps the calculation of effective locking bandwidth of an injection-locked system. Moreover it can be deduced that due to the presence of the sine function, the inequality is limited to the range of:

$$
|\Delta \omega| \leq k_{c} \sqrt{\frac{S_{\text {inj }}}{S}}\left[\sqrt{1+\alpha_{H}^{2}}\right]
$$

On the other hand, it appears that the oscillation limit for $\theta$ is between $-\pi / 2$ and $\pi / 2 . \Delta \omega$ is then bounded by:

$$
-k_{c} \sqrt{\frac{S_{i n j}}{S}}\left[\sqrt{1+\alpha_{H}^{2}}\right] \leq \Delta \omega \leq k_{c} \sqrt{\frac{S_{i n j}}{S}}
$$

The asymmetry of the locking range can be explained both mathematically and physically. Mathematically speaking, if we observe (14), we can see that due to the multiplication with the term $\alpha_{H}$ on the left hand side, this relation becomes asymmetric with respect to $\alpha_{H}$. 


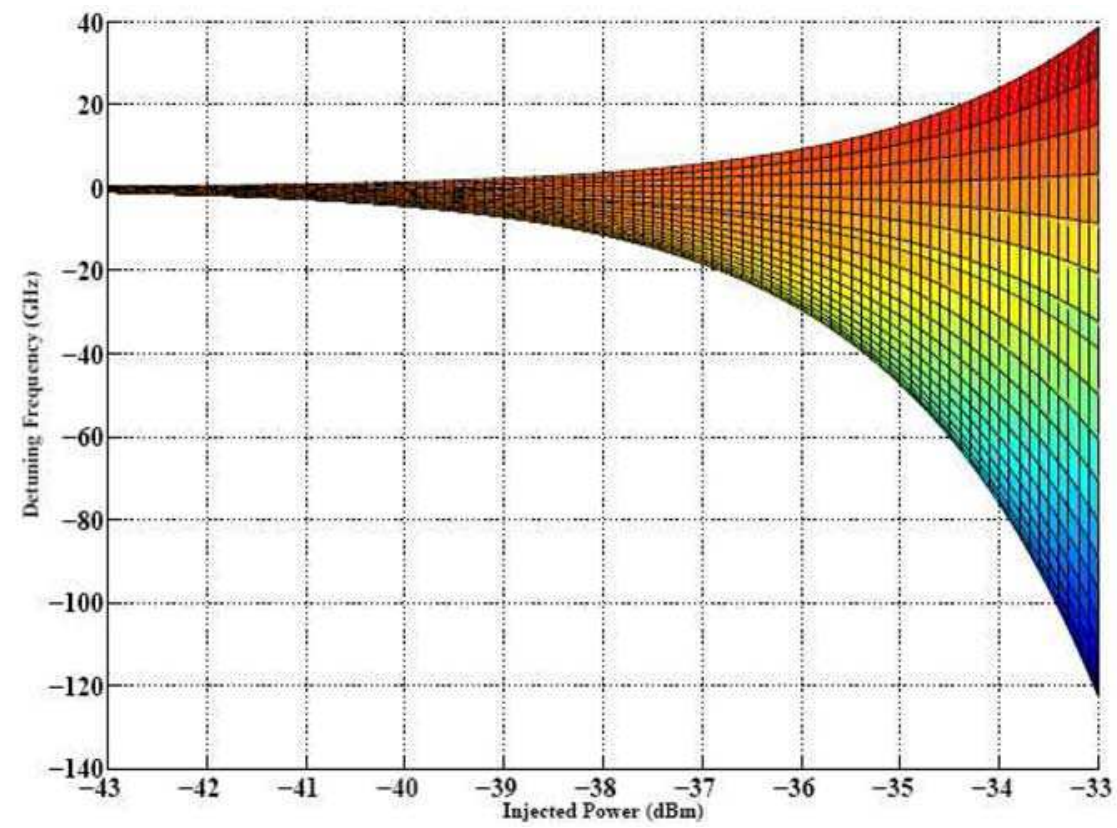

Fig. 7. 2D presentation of calculated locking range of a long wavelength VCSEL with $\alpha_{H}=3$ showing the locking-range dependence on injected optical power.

Physically speaking, during the injection-locking of a semiconductor laser the increased photon population changes the refractive index and leads to a cavity wavelength shift in the longer wavelength direction and finally an asymmetric locking range. Calculated lockingrange for $\alpha_{H}=3$ is presented in fig. 7. It can be observed from equation (14) that a higher value of $\alpha_{H}$ leads to higher locking-range: A higher value of $\alpha_{H}$ favours locking in the negative frequency detuning range. In terms of locking-range characteristics, VCSELs are different from EELs. Locking range determines the extent of frequency enhancement of an optically injection-locked laser. Equation (14) shows that the locking-range depends on injected power and coupling coefficient $\mathrm{k}_{\mathrm{c}}$. Therefore mathematically it can be stated that the locking-range follows the variation of the term $\mathrm{k}_{\mathrm{c}} \sqrt{S_{i n j} / s}$.

Since a VCSEL cavity is much shorter than an EEL cavity, VCSELs have typically very high values of $k_{c}(10)$ as compared to those of conventional lasers. This implies that VCSEL locking-ranges are higher compared to EEL locking-ranges and can potentially lead to much higher resonance frequencies.

\subsection{Small signal analysis}

We begin by presenting once again the "Modified VCSEL Rate Equations". The small signal analysis is performed to derive the $S_{21}$ response of an injection-locked VCSEL. Consider that a sinusoidal signal modulates a laser biased at current I. The resulting expression for current I then becomes:

$$
I(t)=\bar{I}+\Delta I e^{j \omega t}
$$


Similarly, the carrier, photon and phase variations can be described as follows:

$$
\begin{aligned}
N(t) & =\bar{N}+\Delta N e^{j \omega t} \\
S(t) & =\bar{S}+\Delta S e^{j \omega t} \\
\phi(t) & =\bar{\phi}+\Delta \phi e^{j \omega t}
\end{aligned}
$$

By putting

$$
\begin{aligned}
\dot{N} & =\frac{d N}{d t} \\
\dot{S} & =\frac{d S}{d t} \\
\dot{\phi} & =\frac{d \phi}{d t}
\end{aligned}
$$

We have:

$$
\begin{aligned}
& \Delta \dot{N}(I, N, S)=\frac{\partial \dot{N}}{\partial I} \cdot \Delta I+\frac{\partial \dot{N}}{\partial N} \cdot \Delta N+\frac{\partial \dot{N}}{\partial S} \cdot \Delta S \\
& \Delta \dot{S}(N, S, \phi)=\frac{\partial \dot{S}}{\partial N} \cdot \Delta N+\frac{\partial \dot{S}}{\partial S} \cdot \Delta S+\frac{\partial \dot{N}}{\partial \phi} \cdot \Delta \phi \\
& \Delta \dot{\phi}(N, S, \phi)=\frac{\partial \dot{\phi}}{\partial N} \cdot \Delta N+\frac{\partial \dot{\phi}}{\partial S} \cdot \Delta S+\frac{\partial \dot{\phi}}{\partial \phi} \cdot \Delta \phi
\end{aligned}
$$

The gain, as defined in (3), contains both the carrier and the photon terms. Partial differentiation of (3), with respect to the carrier and photon densities $\mathrm{N}$ and $\mathrm{S}$, yields two new variables $G_{N}$ and $G_{S}$, where $G_{N}$ and $G_{S}$ are defined as:

$$
\begin{gathered}
G_{N}=\frac{\partial G}{\partial N}=\frac{a_{0}}{1+\epsilon S} \\
G_{S}=-\frac{\partial G}{\partial S}=\frac{a_{0} \epsilon\left(N-N_{t r}\right)}{(1+\epsilon S)^{2}}
\end{gathered}
$$

Differentiating equation (5) with respect to N, S and $\phi$ therefore results in the following set of three equations:

$$
\begin{gathered}
\frac{\partial \dot{N}}{\partial N} \cdot \Delta N=\left(-\left(A+2 B N+3 C N^{2}\right)-v_{g} G_{N} S\right) \Delta N \\
\frac{\partial \dot{N}}{\partial S} \cdot \Delta S=\left(-v_{g} G+v_{g} G_{S} S\right) \Delta S \\
\frac{\partial \dot{N}}{\partial I} \cdot \Delta I=\frac{\eta_{i}}{q V_{a c t}} \Delta I
\end{gathered}
$$


Similarly if we define a new variable $\rho$ as:

$$
\rho=\frac{v_{g}}{2 L} \sqrt{\frac{S_{i n j}}{S}}
$$

And differentiate equation (6) with respect to $\mathrm{N}, \mathrm{S}$ and $\phi$ we have the following set of equations:

$$
\begin{aligned}
\frac{\partial \dot{S}}{\partial N} \cdot \Delta N & =\left(\Gamma v_{g} G_{N} S-2 \beta B N\right) \Delta N \\
\frac{\partial \dot{S}}{\partial S} \cdot \Delta S & =\left(-\Gamma v_{g} G_{S} S+\rho \cos (\theta)\right) \Delta S \\
\frac{\partial \dot{S}}{\partial \phi} \cdot \Delta \phi & =(-2 \cdot \rho S \sin (\theta)) \Delta \phi
\end{aligned}
$$

The partial differentiation of the phase equation (7) with respect to $N, S$ and $\phi$ results in the following set of equations:

$$
\begin{aligned}
\frac{\partial \dot{\phi}}{\partial N} \cdot \Delta N & =\frac{\alpha_{H} \Gamma v_{g} a_{0}}{2} \Delta N \\
\frac{\partial \dot{\phi}}{\partial S} \cdot \Delta S & =\frac{\rho \sin (\theta)}{2 S} \Delta S \\
\frac{\partial \dot{\phi}}{\partial \phi} \cdot \Delta \phi & =-\rho \cos (\theta) \Delta \phi
\end{aligned}
$$

Linearised rate equations can then be expressed as:

$$
\begin{gathered}
\Delta \dot{N}=\frac{\eta_{i}}{q V_{a c t}} \Delta I-\left(A+2 B N+3 C N^{2}+v_{g} G_{N} S\right) \Delta N-\left(v_{g} G-v_{g} G_{S} S\right) \Delta S \\
\Delta \dot{S}=\left(\Gamma v_{g} G_{N} S-2 \beta B N\right) \Delta N-\left(\Gamma v_{g} G S-\rho \cos (\theta)\right) \Delta S-(2 \cdot \rho S \sin (\theta)) \Delta \phi \\
\Delta \dot{\phi}=\left(\frac{\alpha_{H} \Gamma v_{g} a_{0}}{2}\right) \Delta N+\left(\frac{\rho \sin (\theta)}{2 S}\right) \Delta S-(\rho \cos (\theta)) \Delta \phi
\end{gathered}
$$

Replacing the partial derivatives by intermediate variables gives

$$
\begin{aligned}
\Delta \dot{N} & =\frac{\eta_{i}}{q V_{a c t}} \Delta I-\gamma_{N N} \Delta N-\gamma_{N S} \Delta S \\
\Delta \dot{S} & =\gamma_{S N} \Delta N-\gamma_{S S} \Delta S-\gamma_{S \Phi} \Delta \phi \\
\Delta \dot{\phi} & =\gamma_{\Phi N} \Delta N+\gamma_{\Phi S} \Delta S-\gamma_{\Phi \Phi} \Delta \phi
\end{aligned}
$$


This can be readily arranged into a three equation matrix system as follows:

$$
\frac{d}{d t}\left[\begin{array}{c}
\Delta S \\
\Delta N \\
\Delta \Phi
\end{array}\right]=\left[\begin{array}{ccc}
-\gamma_{S S} & \gamma_{S N} & -\gamma_{S \Phi} \\
-\gamma_{N S} & -\gamma_{N N} & \gamma_{N \Phi} \\
\gamma_{\Phi N} & \gamma_{\Phi S} & -\gamma_{\Phi \Phi}
\end{array}\right] \cdot\left[\begin{array}{c}
\Delta S \\
\Delta N \\
\Delta \Phi
\end{array}\right]+\frac{\eta_{i}}{q V_{a c t}} \cdot\left[\begin{array}{c}
0 \\
\Delta I \\
0
\end{array}\right]
$$

Taking the Laplace transform of the equation set in order to pass from time-domain to frequency-domain, and arranging, yields:

$$
\left[\begin{array}{ccc}
\gamma_{S S}+j \omega & -\gamma_{S N} & \gamma_{S \Phi} \\
\gamma_{N S} & \gamma_{N N}+j \omega & 0 \\
-\gamma_{\Phi S} & -\gamma_{\Phi N} & \gamma_{\Phi \Phi}+j \omega
\end{array}\right] \cdot\left[\begin{array}{c}
\Delta \widetilde{S} \\
\Delta \widetilde{N} \\
\Delta \widetilde{\Phi}
\end{array}\right]=\frac{\eta_{i} \tilde{I}}{V_{a c t}} \cdot\left[\begin{array}{l}
0 \\
1 \\
0
\end{array}\right]
$$

In order to solve this three-equation matrix system we have to calculate the determinant of the intermediate variable matrix:

$$
\Lambda=\left|\begin{array}{ccc}
\gamma_{S S}+j \omega & -\gamma_{S N} & \gamma_{S \Phi} \\
\gamma_{N S} & \gamma_{N N}+j \omega & 0 \\
-\gamma_{\Phi S} & -\gamma_{\Phi N} & \gamma_{\Phi \Phi}+j \omega
\end{array}\right|
$$

Where

$$
\begin{aligned}
\Lambda & =-j \omega^{3} \\
& -\omega^{2}\left(\gamma_{N N}+\gamma_{S S}+\gamma_{\Phi \Phi}\right) \\
& +j \omega\left(\gamma_{S \Phi} \gamma_{\Phi S}+\gamma_{S S} \gamma_{N N}+\gamma_{N S} \gamma_{S N}+\gamma_{N N} \gamma_{\Phi \Phi}+\gamma_{S S} \gamma_{\Phi \Phi}\right) \\
& +\left(\gamma_{S \Phi} \gamma_{\Phi S} \gamma_{N N}-\gamma_{S \Phi} \gamma_{N S} \gamma_{\Phi N}+\gamma_{S S} \gamma_{N N} \gamma_{\Phi \Phi}+\gamma_{S N} \gamma_{N S} \gamma_{\Phi \Phi}\right)
\end{aligned}
$$

Using the Kramer's rule, the photon density variation can be expressed as:

$$
\Delta \widetilde{S}=\frac{\eta_{i} \tilde{I}}{q V_{a c t}} \cdot \frac{\left|\begin{array}{ccc}
0 & -\gamma_{S N} & \gamma_{S \Phi} \\
1 & \gamma_{N N}+j \omega & 0 \\
0 & -\gamma_{\Phi N} & \gamma_{\Phi \Phi}+j \omega
\end{array}\right|}{\Lambda}
$$

Simplifying equation 1.55 leads to:

$$
\Delta \widetilde{S}=\frac{\eta_{i} \tilde{I}}{q V_{a c t}} \cdot \frac{\left(\gamma_{S N} \gamma_{\Phi \Phi}-\gamma_{\Phi N} \gamma_{N \Phi}\right)+j \omega \gamma_{S N}}{\Lambda}
$$

(6) and (7) can alternatively be solved to obtain a relation in terms of the phase difference between two lasers and is presented below:

$$
\theta=\sin ^{-1}\left[\frac{\Delta \omega}{k_{c} \sqrt{\frac{S_{\text {inj }}}{S}} \sqrt{1+\alpha_{H}^{2}}}+\tan ^{-1} \alpha_{H}\right]
$$




\subsection{Numerical simulations}

The mathematical model proposed above is implemented in MATLAB in order to observe the small-signal response of an injection-locked system. Table 1 summarises the VCSEL intrinsic parameters used to calculate the $S_{21}$ response of an injection locked system (Bacou, 2008).

\subsection{Simulation results}

Recently the most significant application of optical injection-locking has been in the domain of resonance frequency enhancement. The enhanced resonance frequency can lead to an extended bandwidth many times the original device bandwidth. The modulation response of an injection-locked laser can be characterized as one of the following three:

- High Resonance Frequency, Low Bandwidth

- High Resonance Frequency, High Bandwidth

- Low Resonance Frequency, Low Bandwidth

Although the resonance frequency of an optically injection-locked laser increases with increasing injected power levels, the frequency detuning between the two lasers plays a very important role in determining the eventual characteristics of the $S_{21}$ curve and finally the effective bandwidth. The above presented three different kinds of modulation responses depend on different locking conditions and parameters and are described in the following section.

\subsection{High resonance frequency, low bandwidth}

The high resonance frequency, low bandwidth operation regime can be attributed to a positive frequency detuning. Since the resonance frequency of an injection-locked system is the difference between the master laser frequency and the down shifted follower cavity frequency, positive frequency detuning results in very high resonance frequencies.

\begin{tabular}{|c|c|c|}
\hline Parameter & Units & Value \\
\hline$\eta_{i}$ & - & 0.8 \\
\hline$\tau_{e}$ & $n s$ & 0.61 \\
\hline$N_{t h}$ & $\mathrm{~cm}^{-3}$ & $5.33 \times 10^{18}$ \\
\hline$N_{t r}$ & $\mathrm{~cm}^{-3}$ & $3.24 \times 10^{18}$ \\
\hline $\mathrm{A}$ & $\mathrm{s}^{-1}$ & $1.1 \times 10^{8}$ \\
\hline $\mathrm{B}$ & $\mathrm{cm}^{3} / \mathrm{s}$ & $1 \times 10^{-10}$ \\
\hline $\mathrm{C}$ & $\mathrm{cm}^{6} / \mathrm{s}$ & $3.57 \times 10^{-29}$ \\
\hline$a_{0}$ & $\mathrm{~cm}^{2}$ & $4.8 \times 10^{-16}$ \\
\hline$v_{g}$ & $\mathrm{~cm}^{2} \mathrm{~s}$ & $7.7 \times 10^{9}$ \\
\hline$\epsilon$ & $\mathrm{cm}^{3}$ & $2.2 \times 10^{-17}$ \\
\hline$\tau_{P}$ & $p s$ & 6.4 \\
\hline$S$ & $\mathrm{~cm}^{-3}$ & $2.5 \times 10^{15}$ \\
\hline
\end{tabular}

Table 1. Long wavelength VCSEL intrinsic parameters used to simulate the small-signal injection-locking behaviour (Bacou, 2008). 


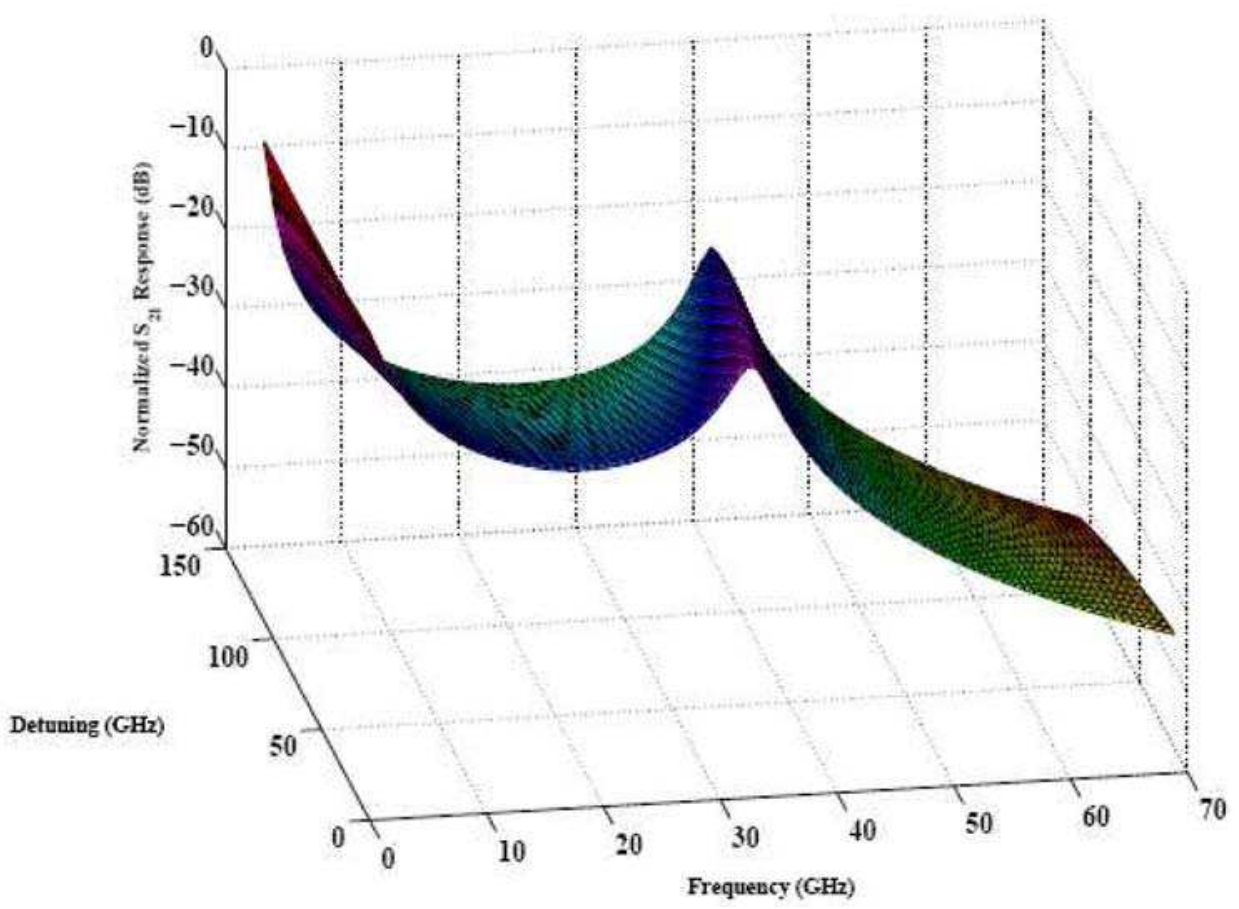

Fig. 8. Calculated $\mathrm{S}_{21}$ response of an optically injection-locked VCSEL with constant injected power and variable positive frequency detuning. The detuning is varied from $10 \mathrm{GHz}$ to 110 $\mathrm{GHz}$.

On the other hand, optically injection-locked systems can be mathematically defined as third-order systems and suffer from low-frequency dips due to the presence of a parasitic pole. Fig. 8 presents the simulated $S_{21}$ response of an optically injection-locked VCSEL operating in the positive frequency detuning regime. The injected optical power is maintained constant for this set of curves in order to study the effects of variation in positive frequency detuning. The resonance frequency increases with increasing difference between the master and follower VCSEL frequencies.

Although from a telecommunication point of view, enhancement in resonance frequency is desired but the low frequency dip of an optically injection-locked system operating in the positive frequency detuning regime limits the effective bandwidth of the system and renders the system inefficient. This configuration therefore is not desired for operation in Datacom and telecommunication environments.

Very high resonance frequencies however can be beneficial for another very important application i.e. the generation of millimetre-wave signals. Since the proposal of the $60 \mathrm{GHz}$ band for the radio link frequency in broad-band cellular systems, the utilization of optical fibre for signal distribution has attracted much interest. This is due to low-loss nature of the optical fibres that are capable of transmitting data at very high bit rates. The main obstacle in the implementation of this scheme is the conception of a high frequency oscillator. Goldberg et. al had already demonstrated the generation of microwave signals using 
injection-locked laser diodes in 1983 [16], but the enthusiasm in the implementation of this scheme faded away due to the incipient nature of semiconductor lasers at that time.

\subsection{High resonance frequency, high bandwidth}

Positive frequency detuning can be employed to achieve very high resonance frequencies that could be useful for certain applications such as microwave and millimetre-wave signal generation but such high resonance frequencies imply very low cut-off frequencies due to low-frequency dip associated to positive frequency detuning. This situation can be improved by operating the laser at close to zero detuning. In such a configuration, the cutoff frequency increases with increase in injected power but due to very low frequency detuning value there is no loss at low frequency values. Frequency detuning has little or no effect on the resonance frequency of such a system and the bandwidth increase is dependent only on optical injected power. This configuration can be employed for broadband digital communications that require the transmission of very high bit rates. The third operation regime is defined by negative frequency detuning. Fig. 2.10 presents a set of simulated $S_{21}$ curves with increasing negative frequency detuning. It is clear from Fig. 2.10 that for positive frequency detuning values, the follower VCSEL $S_{21}$ response is un-damped with high resonance frequencies. However when the detuning between the two VCSELs is varied in the negative detuning operation regime, the $S_{21}$ response curves start to become highly damped.

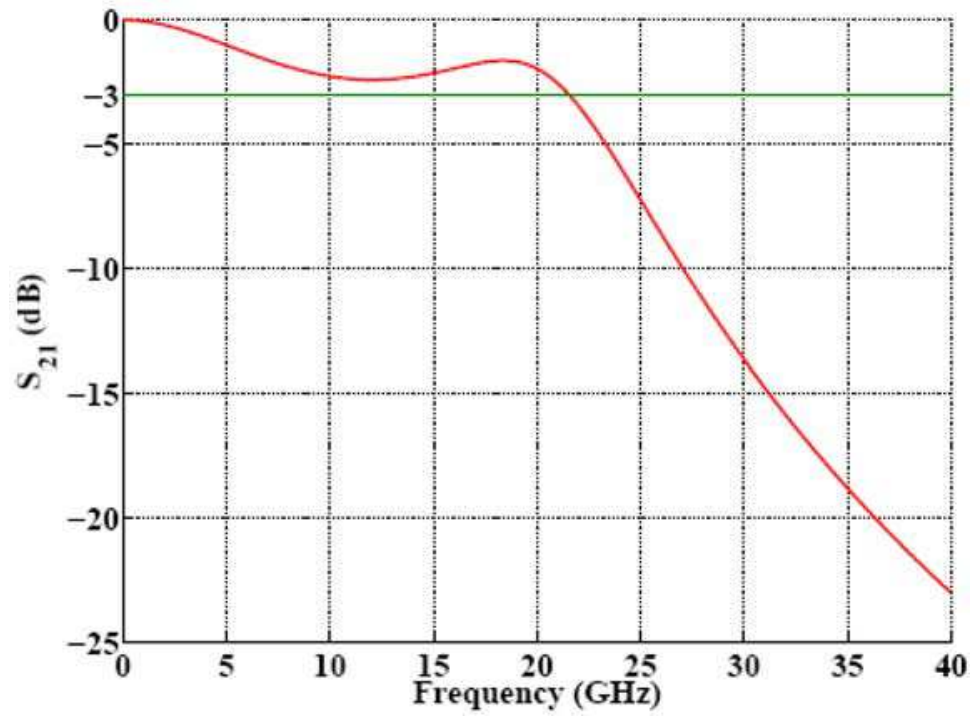

Fig. 9. Simulated $S_{21}$ response of an optically injection-locked follower VCSEL showing cutoff frequency enhancement.

\subsection{Low resonance frequency, low bandwidth}

At the same time, the low frequency dip, exhibited due to positive frequency detuning operation regime starts to disappear. Finally at relatively high values of negative frequency detuning the $S_{21}$ curves become over-damped and gradually the resonance peak vanishes. 


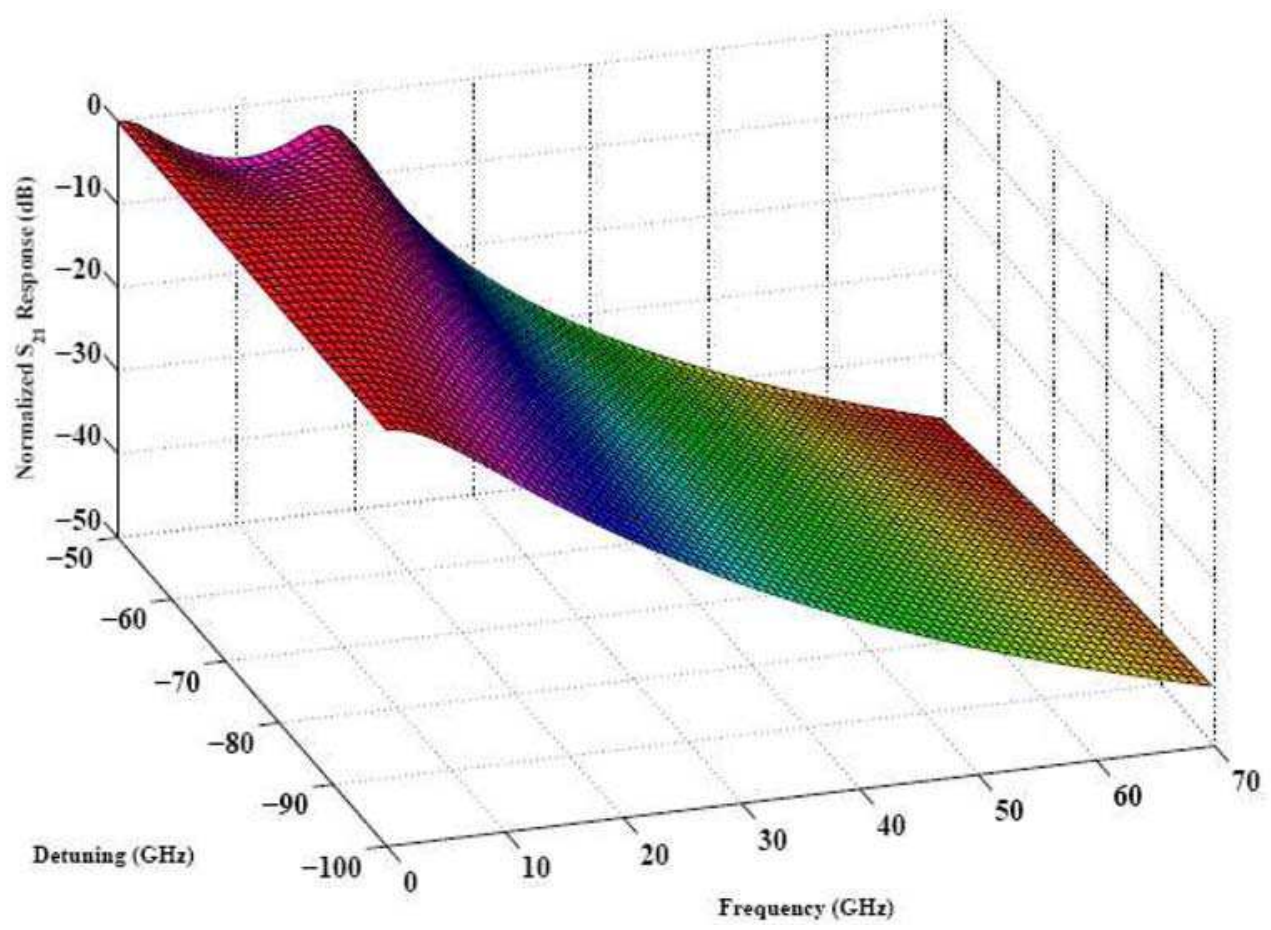

Fig. 10. Calculated $S_{21}$ response of an optically injection-locked VCSEL with constant injected power and variable negative detuning. The detuning is varied from $10 \mathrm{GHz}$ to $-190 \mathrm{GHz}$.

The negative frequency detuning can hence be used to generate high low frequency gain $S_{21}$ curves. This is particularly important for directly modulated optical fibre links. The losses in such links, apart from coupling and connector losses, are due to Electrical- Optical (E/O) and Optical-Electrical (O/E) conversion. Sung et al. have demonstrated that by injectionlocking a laser in negative frequency detuning regime the RF link gain can be improved by up to $10 \mathrm{~dB}$ [21].

\subsection{Comparison between free-running and injection-locked VCSEL models}

Fig. 11 presents a comparison between the free-running and injection-locked $S_{21}$ response of a VCSEL. The frequency responses are plotted on a logarithmic scale in order to highlight the difference between the respective slopes of the two systems. The injection-locked system has a slope of $-18 \mathrm{~dB} /$ octave as compared to a slope of $-12 \mathrm{~dB} /$ octave for a free-running VCSEL.

Another important difference of note is the low frequency dip in the optically injectionlocked VCSEL $S_{21}$ response which is due to the extra pole in the transfer function denominator. By putting $S_{\text {inj }}$ and $\Delta \omega$ to zero the modified VCSEL rate equations are reduced to classical VCSEL rate equations.

The simulations, under different operating conditions, of optically injection-locked VCSELs presented in this chapter reveal certain interesting patterns. First of all, it must be noted that due to the very highly selective nature of the DBR mirrors used in the VCSEL 
manufacturing, a very small amount of light enters in the cavity. This is clear from the locking-range calculations presented in Fig. 7. It is therefore not the injected optical power intensity that is mainly responsible for injection-locked VCSELs' $S_{21}$ curves variations. It is in fact the coupling factor $k_{c}$ whose numerical value is responsible for high locking ranges, facility of injection-locking and high resonance frequencies.

Another important point is the $S_{21}$ curve shape dependence on the frequency detuning value between the two VCSELs. The frequency detuning is the dominant factor in determining the shape of the $S_{21}$ curve and whether it would be high resonance frequency under-damped response or a low resonance frequency high bandwidth flat response. This phenomenon can be explained by understanding the beat-frequency generation effect produced inside the follower VCSEL optical cavity.

Finally, due to optical coupling with the master laser, the dynamic response characteristics of the follower VCSEL change. Usually a two-equation mathematical model is utilized in VCSEL dynamic response simulations. This model gives way to a three-equation system which incorporates the effect of external light injection. Due to this third equation, the presence of a 3rd pole is observed in the transfer function of the optically injection-locked VCSEL. At positive detuning frequency values, this pole becomes dominant at low frequencies and causes the $S_{21}$ response to suffer dips of several dBs which in turn severely limits the effective bandwidth of the system.

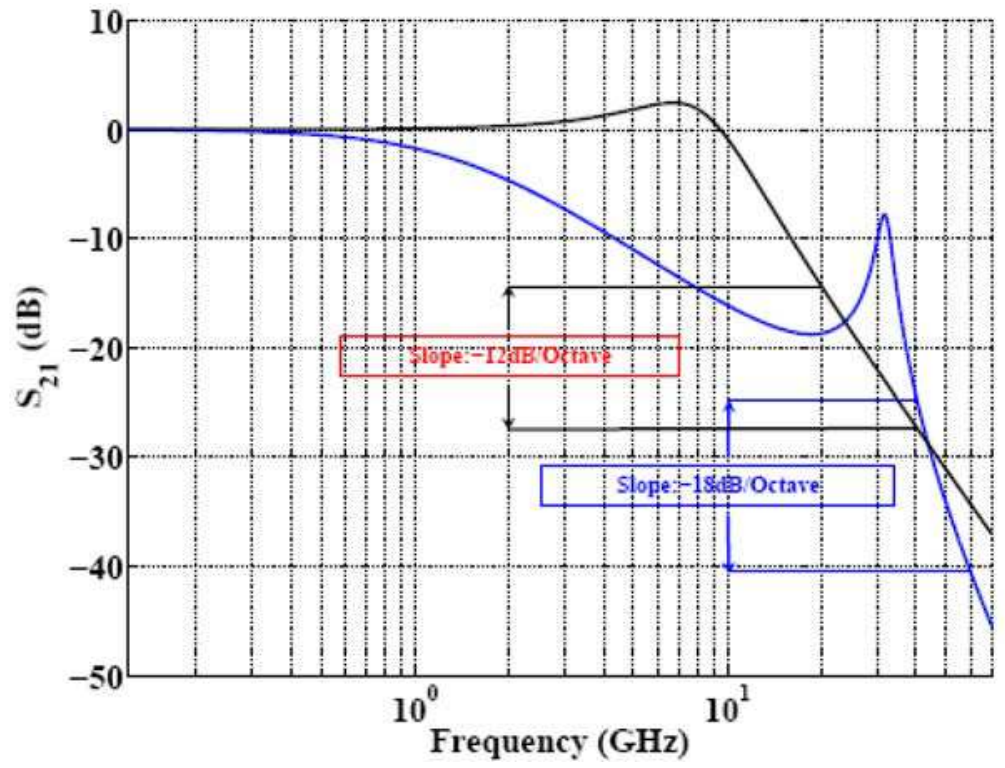

Fig. 11. Comparison between the free-running and injection-locked transfer functions of a VCSEL.

The injection-locking experiments carried-out during the course of this work evolved progressively in their complexity. The objective was to demonstrate and understand the VCSEL-by-VCSEL optical injection-locking phenomena under different operating constraints. Our focus was the study of variations in $S_{21}$ response of injection-locked VCSELs under different injection powers and varying detuning frequencies. 


\section{Experiments using multimode lasers}

\subsection{Multimode Edge Emitting Lasers (EELs)}

Optically injection-locked lasers are known to overcome many fundamental limitations of free-running systems. One of the very important improvements proposed by the employment of the optical injection-locking technique is the side-mode suppression of a multimode laser (Iwashita and Nakagawa, 1982). Fig. 12 presents the superimposed optical spectra of a free-running and an injection-locked laser diode. The Fabry-Pérot modes, visible in the free-running regime, undergo approximately $35 \mathrm{~dB}$ suppression when injectionlocked using a DFB laser diode.

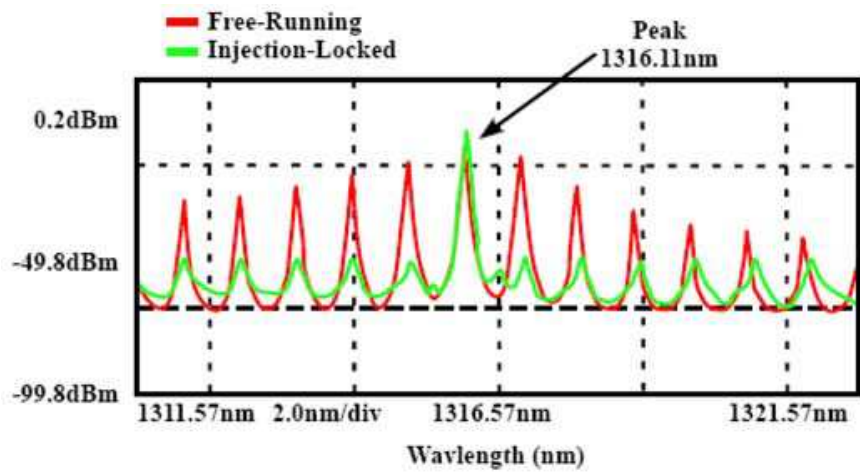

Fig. 12. The super-imposed spectra of a free running and an injection locked Fabry-Pérot EEL. Mode suppression can be observed in the injection locked spectrum.

In the stable locking regime the follower laser frequency is locked to the master laser lasing frequency. The injection-locked Fabry-Pérot mode therefore becomes dominant and the unlocked modes are suppressed. Iwashita et. al demonstrated the utilization of this method for the suppression of mode-partition noise [1]. The employment of optical-injection locking for side-mode suppression in VCSELs however is not very effective. This is due to the difference in the side-mode generation mechanism between the EELs and the VCSELs. A detailed analysis of side-mode generation is presented in the following section.

Single-mode operation of the follower laser however is highly desirable due to another very important reason. As presented in figure 3.2, the locking-range of an injection-locked laser, in the "stable operation region", is dependent on the injected optical power. This effective locking-range is exploitable only if the follower laser is single-mode. If the follower laser is multimode, the achievable detuning frequency is limited by the Free Spectral Range (FSR) of the follower laser. At large detuning frequencies, the master laser might come closer to an adjacent longitudinal mode and in that case, it will lock the adjacent longitudinal mode instead of sweeping the entire locking range with previously locked mode. This modehopping reduces the effective "locking" and hence "operation range" of an injection-locked system.

\subsection{Multimode VCSELS}

Fig. 13 presents the optical spectrum of a multimode VCSEL. The VCSEL in question is manufactured by Vertilas with a threshold current of $6 \mathrm{~mA}$ and peak output optical power 
of $20 \mathrm{~mW}$. The VCSEL chip was powered-up using a probe-station. The master laser is single-mode Vertilas VCSEL emitting in the $1.55 \mu \mathrm{m}$ range. A comparison with Fig. 14 shows that optical injection-locking fails to produce an effect similar to that demonstrated previously on multimode EELs. Although nominal side-mode suppression is observed in the injection-locked follower VCSEL spectrum, the emission spectrum rests multimode.

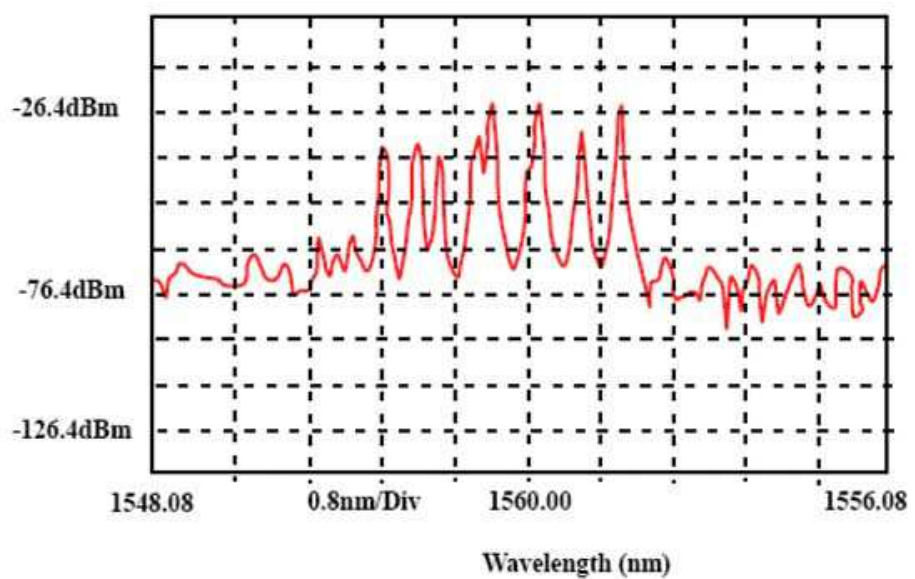

Fig. 13. Optical spectrum of an Vertilas multimode "Power" VCSEL. The VCSEL threshold current is about $6 \mathrm{~mA}$.

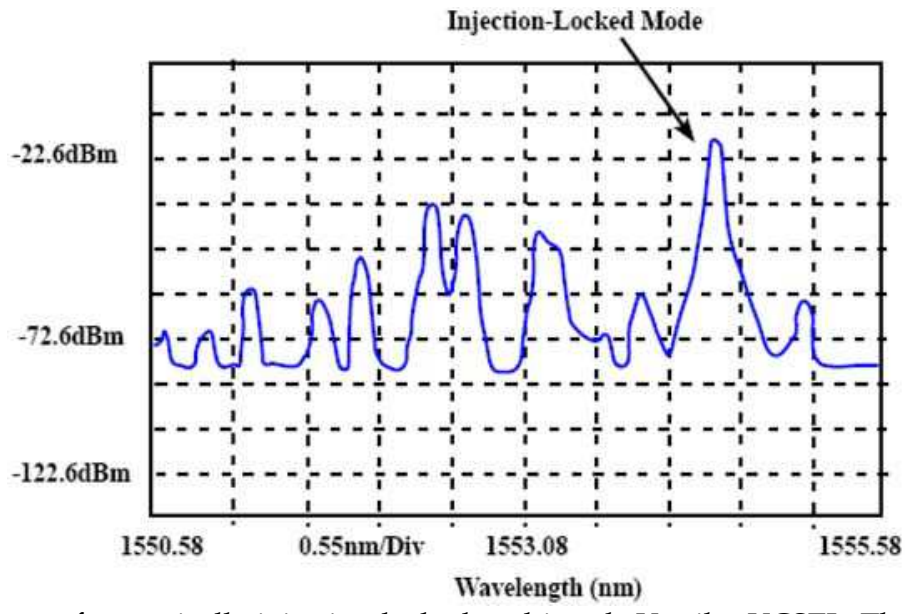

Fig. 14. Spectrum of an optically injection-locked multimode Vertilas VCSEL. The threshold current is about $6 \mathrm{~mA}$. Very feeble side-mode suppression is observed due to injection-locking.

\subsection{Experiments using single-mode VCSELs}

This can be explained by developing an understanding of the side-mode generation phenomena in VCSELs. The active region of a VCSEL is very short as compared to that of an EEL, essentially of the order of the emission wavelength. Consequently, only one FabryPérot mode exists in the VCSELs, since the physical dimensions of the cavity eliminate the 
possibility of longitudinal multi-mode lasing action. Therefore VCSELs are fundamentally single-mode emission devices. However, the confinement and guiding of the optical field thus generated is made very difficult due to a very peculiar VCSEL structural characteristic.

VCSEL design suggests the sharing of a common path for photons and carriers, moving through the DBRs. This leads to the heating of the DBRs due to carrier flow and results in a variable refractive index distribution inside the VCSEL optical cavity. The creation of nonuniform refractive index zones inside the optical cavity leads to different optical paths and has an overall dispersive effect. This phenomenon is known as "Thermal Lensing".

The electrons passing through the DBRs tend to concentrate on the edge of the active zone due to the oxide aperture-based carrier guiding. A higher carrier concentration at the fringes of the active zone translates into higher photon generation at the edges of the active zone. Instead of being concentrated in the centre of the optical cavity, in the form of a single transverse mode, the optical energy is repartitioned azimuthally inside the optical cavity. The creation of non-uniform refractive index zones within the VCSEL optical cavity, changes the effective optical path inside the cavity which manifests itself in the form of undesired side-modes. Since the VCSEL sidemodes are a consequence of spatial energy distribution, they are referred to as "Spatial" or "Transverse Modes". Higher bias currents therefore imply high optical power and in consequence a higher number of transverse modes. An oxide-aperture is employed in order to achieve optimal current confinement and to block unwanted transverse modes. The oxide-aperture diameter determines the multimode or single mode character of a VCSEL. VCSELs having oxide aperture diameters greater than $5 \mu \mathrm{m}$ exhibit a multimode behaviour.

It can also be inferred from the above discussion that for the type of VCSELs employing the oxide-aperture technology for optical confinement, single mode VCSELs almost always have emission powers less than those of multimode VCSELs. Since the Vertilas VCSEL used here is a high power device, it has a Buried Tunnel Junction (BTJ) diameter of $20 \mu \mathrm{m}$ and is therefore distinctly multimode. Since optical injection-locking favours single-mode operation by eliminating longitudinal modes and since the modes generated in VCSELs are not longitudinal, the employment of optical injection-locking for single-mode VCSEL operation is not very effective.

\subsection{Experiments using vertilas VCSELs}

A logical step, after trying optical injection-locking of multimode VCSELs, was to attempt the injection-locking of single-mode VCSELs. The VCSELs used for initial injection-locking experiments were manufactured by Vertilas $\mathrm{GmbH}$. These are single-mode, TO-46 packaged, pigtailed, Buried Tunnel Junction (BTJ) devices with an emission wavelength of $1.55 \mu \mathrm{m}$. The L-I curve of the follower VCSEL is presented in figure 3.5 (a). The mode suppression ratio between the fundamental and the side-mode is approximately $40 \mathrm{dBs}$. The injection-locking experiments using Vertilas VCSELs were simple to carry-out due to the pigtailed nature of the components that made the optical power-injection inside the follower VCSEL cavity relatively easy. The well known phenomenon of sidemode suppression (as demonstrated with EELs and presented in figure 12) was observed. When the VCSEL satellite mode is optically injection-locked, the fundamental mode undergoes a rapid diminution and the VCSEL output optical power shifts to the side-mode wavelength. However, other than being a proof of concept demonstration, this exercise proved to be of little significance. The real price of this ease of manipulation was paid in terms of a degraded frequency response. 
The TO-46 package cut-off frequency was about $5 \mathrm{Ghz}$ which was well below the component cut-off frequency $(11 \mathrm{GHz})$. The observation of injection-locked VCSELs' $\mathrm{S}_{21}$ response under various injection conditions was therefore not possible.

\subsection{Experiments using RayCan VCSELs}

The optically injection-locked follower VCSEL $S_{21}$ responses presented above provide very interesting results. Especially the availability of on-chip components allows the observation of parasitics-free free-running and injection-locked $S_{21}$ responses. It was noticed however that the Master VCSEL is not modulated for these injection-locking experiments and hence needs not be on-chip.

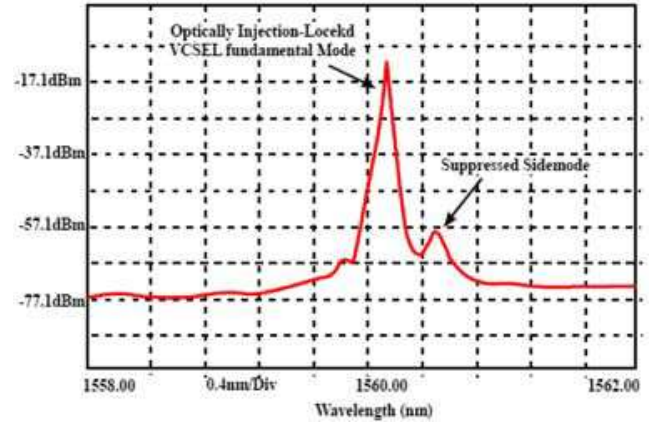

(a)

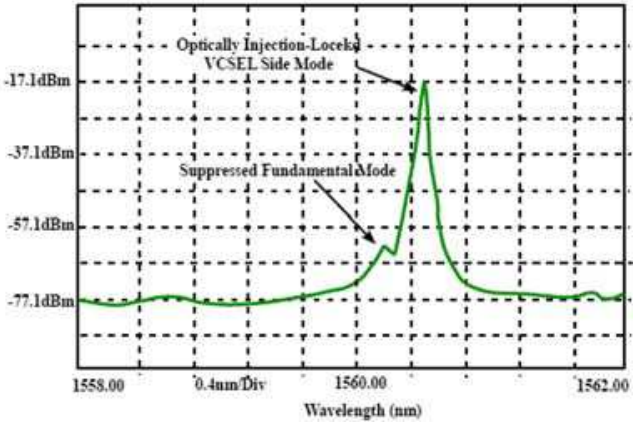

(b)

Fig. 15. (a) Optical spectrum of an optically injection-locked Vertilas VCSEL. The locking of fundamental mode further suppresses the side-mode. (b) Optical spectrum of an optically injection-locked Vertilas VCSEL. The locking of side mode has suppressed the fundamental lasing mode. Notice the position of the suppressed modes in the two different cases.

The employment of a fibred master VCSEL will facilitate the injection-locking experiments in the following ways:

- This will allow the utilization of only one probe-station instead of two thus reducing the test-bench size and minimizing its complexity.

- This will increase the magnitude of available optical power since the coupling losses on the master VCSEL side would be eliminated.

Also, injection-locking experiments in the static domain such as linewidth, polarization and RIN measurements could be carried out using fibred follower VCSEL without suffering from packaging parasitics performance penalties. It was then decided to carry-out injectionlocking experiments using commercially available RayCan VCSELs.

\subsection{RayCan VCSELs structure}

The structure of a 1.3 $\mu \mathrm{m}$ RayCan VCSEL is presented in Fig. 6. RayCan VCSELs are bottomemitting type, as has been explained above. As far as the incorporation of a bottom-emitting VCSEL in an optical sub-assembly is concerned, the application of normal integration techniques such as wire-bonding or flip-chip designs is easily applicable. However, probestation testing of bottom-emitting components poses some challenging problems. Bottomemission implies the existence of electrodes on the reverse side of the VCSEL chip, as shown in figure 3.20. This means that in order to power-up the VCSEL, using coplanar probes, the chip has to be inverted. 


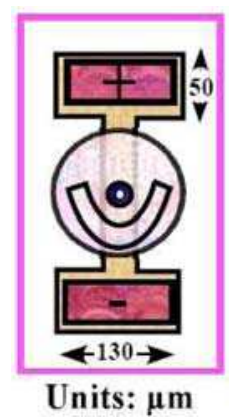

Fig. 16. Bottom-emitting on-chip RayCan VCSEL with 1.3um operation wavelength.

The chip-inversion, in turn, implies the impossibility of optical power collection with a single-mode or multimode fibre. On the other hand, if the chip is used in the top-emitting configuration, it becomes impossible to power-up the chip using probes.

Another problem was the distance between the two electrodes. The probes used for VCSEL testing have a pitch of $125 \mu \mathrm{m}$. However the distance between the two RayCan VCSEL electrodes is about $300 \mu \mathrm{m}$. Without using $300 \mu \mathrm{m}$ pitch probes, it would have been impossible to power-up the VCSELs anyway. These two problems were solved by getting the VCSEL chip integrated to a sub-mount. The sub-mount was prepared by RayCan for VCSEL integration with a monitoring photodiode, inside a TO-46 package. As per our demand, the VCSEL chips were integrated to the sub-mounts and delivered to us unpackaged. Furthermore, the intent of optical injection-locking experiments was observation of the enhanced $S_{21}$ response. This objective was compromised by the employment of the sub-mount, as the $S_{21}$ response was limited by the parasitic transmission line frequency.

The presence of air-gaps in the VCSEL structure implies lower intrinsic cut-off frequencies. The inevitable utilization of the sub-mount assembly, combined with the above-mentioned structural deficiency, renders these VCSELs relatively low frequency operation devices. It is perhaps due to this reason that the 10 Gbps modules supplied by RayCan employ four VCSELs in parallel configuration to achieve 10Gbps bit rate, as opposed to Vertilas 10Gbps modules that are composed of only one VCSEL.

\subsection{Injection locking experiments}

The availability of fibred components however simplified the test-bench considerably. In stead of using two probe-stations for master and follower VCSELs respectively, only one probe-station was used since only the follower VCSEL was used in the on-chip configuration.

The utilization of a pigtailed master VCSEL also increased the available optical power and allowed the elimination of the OSA from the injection-locking setup. Fig. 17 presents the optical injection-locking test-bench used for RayCan VCSEL experiments schematically. The utilization of a pigtailed master VCSEL made the testbench considerably compact and increased the available optical power but despite these advantages, the follower VCSEL injection-locked $S_{21}$ spectra do not exhibit very large resonance frequencies. Fig. 18 presents the $S_{21}$ response of an optically injection-locked RayCan follower VCSEL, in the positive frequency detuning regime. Compared to the free-running responses presented, it is clear that an 
increased resonance frequency is observed. Also, due to operation in the positive frequency detuning regime, the $S_{21}$ is un-damped and therefore the resonance peak is very pronounced.

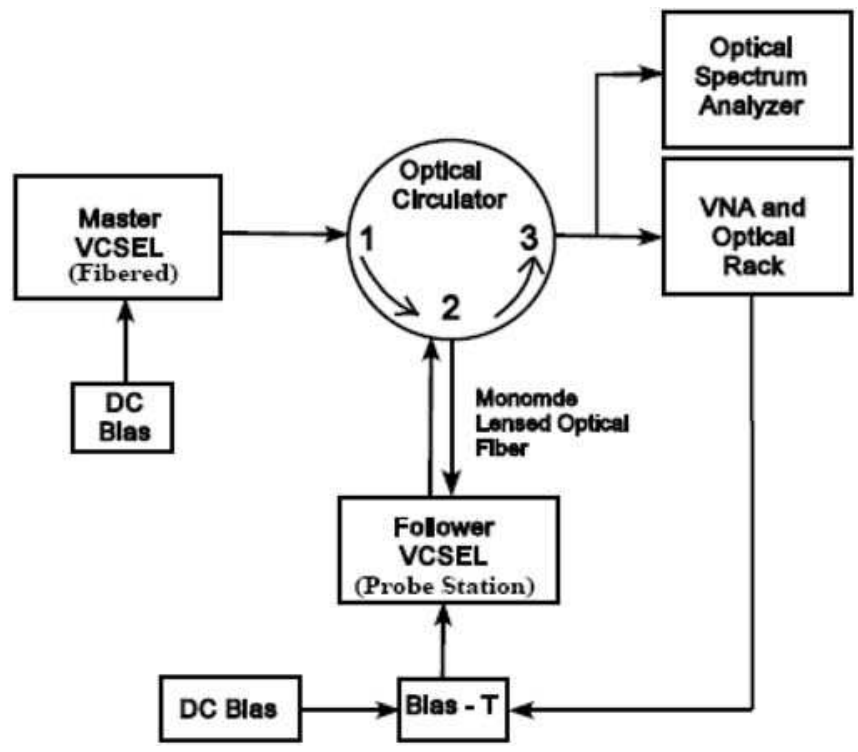

Fig. 17. Schematic representation of the test-bench employed for injection-locking experiments using RayCan VCSELs emitting at $1.3 \mu \mathrm{m}$.

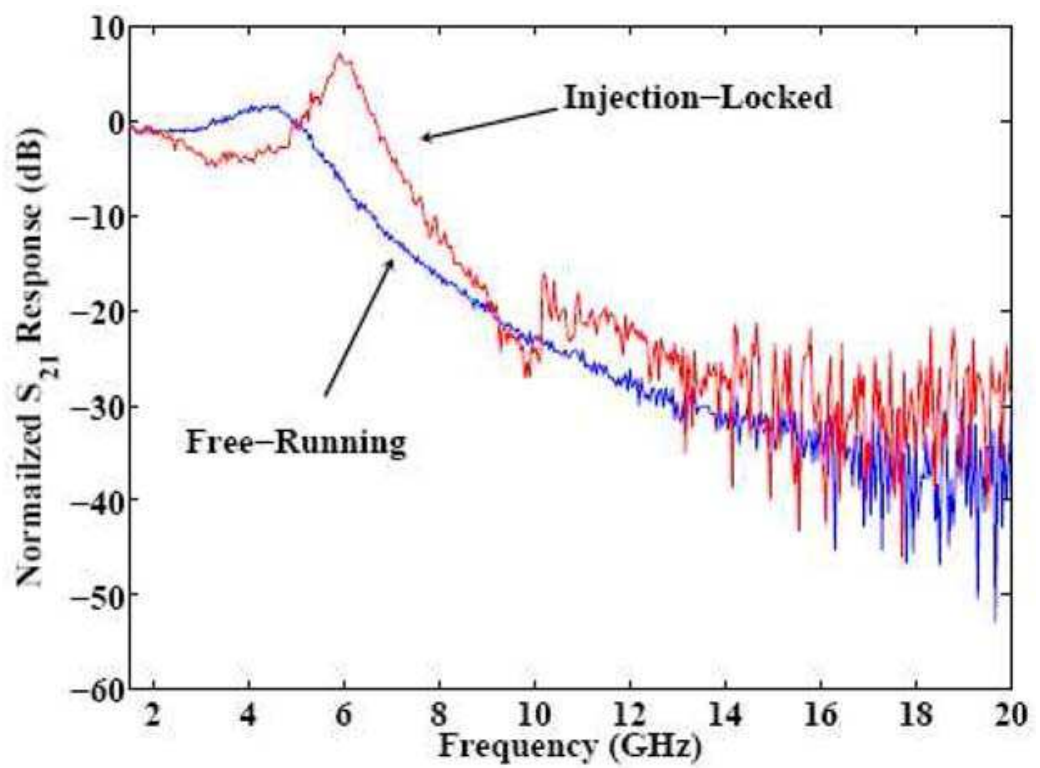

Fig. 18. $S_{21}$ response of an optically injection-locked RayCan VCSEL emitting at $1.3 \mu \mathrm{m}$ operating in the positive frequency detuning regime. 


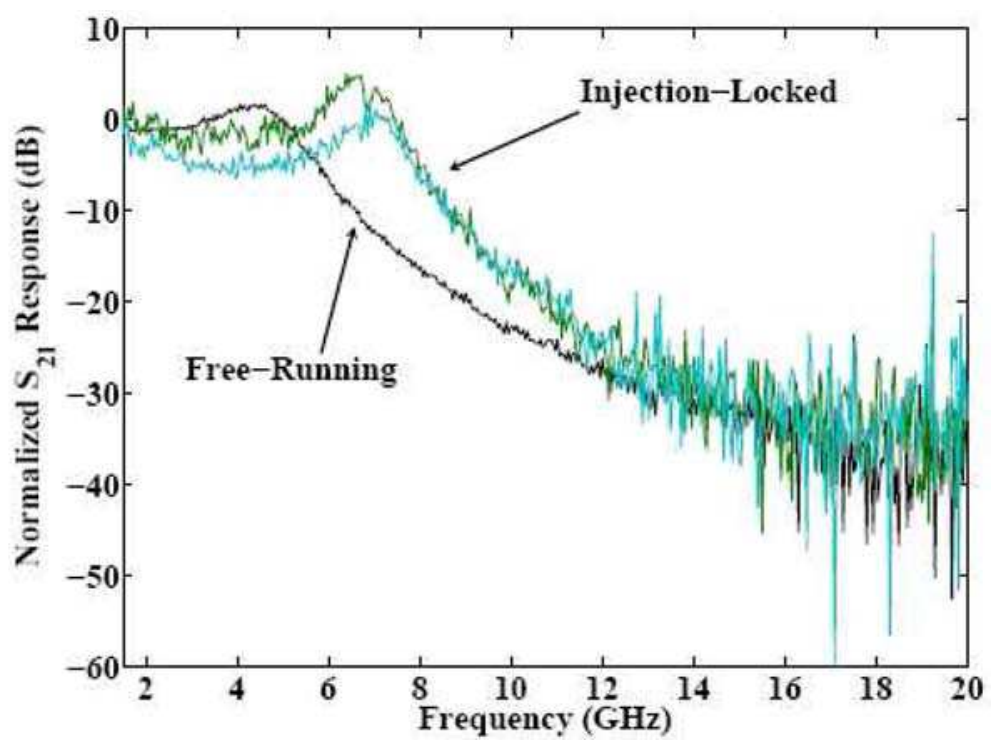

Fig. 19. $S_{21}$ response of an optically injection-locked RayCan VCSEL emitting at $1.3 \mu \mathrm{m}$ operating in the positive frequency detuning regime.

\section{Conclusion and discussion}

Experimental studies of VCSEL-by-VCSEL optical injection-locking phenomena were presented in this chapter. It was demonstrated that optical injection-locking suppresses only the Fabry-Pérot modes of an optical cavity. The transverse modes commonly found in VCSELs remain largely unaffected by optical injection-locking. VCSEL-by- VCSEL optical injection-locking was presented using fibred single-mode VCSELs and fundamental and sidemode suppression phenomena were demonstrated.

Optical injection-locking of on-chip VCSELs was suggested, in order to observe the parasitics free $S_{21}$ response. Three different operation regimes were explored using VCSELby- VCSEL optical injection-locking. Resonance frequencies as high as $7 \mathrm{GHz}$ were presented for follower VCSELs operating in positive frequency detuning regimes. It was however observed that positive frequency detuning increases the resonance frequency but limits the effective bandwidth of the injection-locking system which is not desirable for VCSEL employment in high bit rate telecommunication system.

The zero or slightly negative detuning regime proposes flat, highly damped $S_{21}$ curves. An increase in injected optical power, while remaining keeping the VCSELs in negative detuning configuration, results in the increase of effective bandwidth. Effective bandwidths as high as $10 \mathrm{GHz}$, using optical injection-locking, have been demonstrated. It must be noted that the free-running cut-off frequency of the VCSELs used is about $5 \mathrm{GHz}$. In order to simplify the optical injection-locking setup, the utilization of a fibred master VCSEL has been proposed. Such a configuration also increases the effective available optical power. Optically injection-locked follower VCSEL $S_{21}$ response has been presented in different operating conditions. Experimental results and numerical calculations using the mathematical model have been compared. 


\section{References}

T. Baba, Y. Yogo, K. Suzuki, F. Koyama, and K. Iga, "Near room temperature continuous wave lasing characteristics of GaInAsP/InP surface emitting laser," Electronics Letters, vol. 29, no. 10, pp. 913-914, May 1993.

A. Bacou, A. Rissons, and J.-C. Mollier, "Spectral behavior of long wavelength VCSELs," SPIE Vertical-Cavity Surface-Emitting Lasers XII, vol. 6908, no. 1, 2008.

A. Bacou, "Caractérisation et Modélisation optoélectronique de VCSEL à grande longueur d'onde pour sousensembles optiques intégrés," Ph.D. dissertation, Institut Supérieur de l'Aéronautique et de l'Espace, 2008.

R. L. Batdorf, G. C. Dacey, R. L. Wallace, and D. J. Walsh, "Esaki Diode in InSb," Journal of Applied Physics, vol. 31, no. 3, pp. 613-614, 1960.

A. C. Bordonalli, C. Walton, and A. J. Seeds, "High-Performance Phase Locking of Wide Linewidth Semiconductor Lasers by Combined Use of Optical Injection Locking and Optical Phase-Lock Loop," J. Lightwave Technol., vol. 17, no. 2, p. 328, 1999.

J. Boucart, C. Starck, F. Gaborit, A. Plais, N. Bouche, E. Derouin, L. Goldstein, C. Fortin, D. Carpentier, P. Salet, F. Brillouet, and J. Jacquet, "1-mW CW-RT monolithic VCSEL at 1.55 um," IEEE Photonics Technology Letters, vol. 11, no. 6, pp. 629-631, Jun 1999.

C. A. Burrus, "Indium Phosphide Esaki Diodes," Solid-State Electronics, vol. 5, p. 357, 1962.

C.-H. Chang, L. Chrostowski, C. Chang-Hasnain, and W. Chow, "Study of long wavelength VCSEL-VCSEL injection locking for 2.5-Gb/s transmission," IEEE Photonics Technology Letters, vol. 14, no. 11, pp. 1635-1637, Nov 2002.

C.-H. Chang, L. Chrostowski, and C. Chang-Hasnain, "Injection locking of VCSELs," IEEE Journal of Selected Topics in Quantum Electronics, vol. 9, no. 5, pp. 1386-1393, Sept.Oct. 2003.

L. Chrostowski, C.-H. Chang, and C. Chang-Hasnain, "Reduction of Relative Intensity Noise and Improvement of Spur-Free Dynamic Range of an Injection-Locked VCSEL," The 16th Annual Meeting of the IEEE Lasers and Electro-Optics Society. LEOS 2003, vol. 2, pp. 706-707 vol.2, Oct. 2003.

L. Chrostowski, X. Zhao, and C. Chang-Hasnain, "Microwave Performance of Optically Injection-locked VCSELs," IEEE Transactions on Microwave Theory and Techniques, vol. 54, no. 2, pp. 788-796, Feb. 2006.

L. Chrostowski, F. B., W. Hoffman, M.-C. Amann, S. Wieczorek, and W. Chow, "40 GHz Bandwidth and $64 \mathrm{GHz}$ Resonance Frequency in Injection-Locked $1.55 \mu \mathrm{m}$ VCSELs," IEEE Journal of Selected Topics in Quantum Electronics, Vol. 13, No. 5, 2007.

A. G. Chynoweth, W. L. Feldman, and R. A. Logan, "Excess Tunnel Current in Silicon Esaki Junctions," Physics Review, vol. 121, p. 684, 1961. C. A. Burrus, "Indium Phosphide Esaki Diodes," Solid-State Electronics, vol. 5, p. 357, 1962.

I. Dias, B. Nabet, A. Kohl, J. Benchimol, and J. Harmand, "Electrical and optical characteristics of n-type-doped distributed Bragg mirrors on InP," IEEE Photonics Technology Letters, vol. 10, no. 6, pp. 763-765, Jun 1998.

L. Esaki, "Long journey into tunneling," Proceedings of the IEEE, vol. 62, no. 6, pp. 825-831, June 1974.

M. Espana-Boquera and A. Puerta-Notario, "Noise Effects in Injection Locked Laser Simulation: Phase Jumps and Associated Spectral Components," Electronics Letters, vol. 32, no. 9, pp. 818-819, Apr 1996.

N. M. Froberg and K. Y. Lau, "Ultrahigh-efficiency Microwave Signal Transmission Using Tandem-Contact Single Quantum Well GaAlAs Lasers," in Optoelectronic Signal Processing for Phased-Array Antennas II, vol. 1217, no. 1, 1990, pp. 2-5. 
P. Gallion and G. Debarge, "Influence of amplitude-phase coupling on the injection locking bandwidth of a semiconductor laser," Electronics Letters, vol. 21, no. 7, pp. 264-266, 281985.

P. Gallion, H. Nakajima, G. Debarge, and C. Chabran, "Contribution of spontaneous emission to the linewidth of an injection-locked semiconductor laser," Electronics Letters, vol. 21, no. 14, pp. 626-628, 41985.

M. Grabherr, B. Weigl, G. Reiner, and K. Ebeling, “Comparison of Proton Implanted and Selectively Oxidized Vertical-Cavity Surface-Emitting Lasers," in Conference on Lasers and Electro-optics, 1996. CLEO/Europe., Sep 1996, pp. 165-165.

K. Hasebe, Y. Onishi, and F. Koyama, "Novel polarization controller based on injectionlocked vertical-cavity surface-emitting laser," IEICE Electronics Express, vol. 2, no. 8, pp. 274-279, 2005.

C. Henry, N. Olsson, and N. Dutta, "Locking Range and Stability of Injection Locked $1.54 \mu \mathrm{m}$ InGaAsp Semiconductor Lasers," IEEE Journal of Quantum Electronics, vol. 21, no. 8, pp. 1152-1156, Aug 1985.

N. Holonyak and I. Lesk, "Gallium-Arsenide Tunnel Diodes," Proceedings of the IRE, vol. 48, no. 8, pp. 1405-1409, Aug. 1960.

J. Jewell, J. Harbison, A. Scherer, Y. Lee, and L. Florez, "Vertical-cavity surfaceemitting lasers: Design, growth, fabrication, characterization," IEEE Journal of Quantum Electronics, vol. 27, no. 6, pp. 1332-1346, Jun 1991.

J. Kahn, "1 Gbit/s PSK homodyne transmission system using phase-locked semiconductor lasers," IEEE Photonics Technology Letters, vol. 1, no. 10, pp. 340-342, Oct 1989.

H. Kawaguchi, Y. Yamayoshi, and K. Tamura, "All-Optical Format Conversion Using an Ultrafast Polarization Bistable Vertical-Cavity Surface-Emitting Laser," Lasers and Electro-Optics, 2000. (CLEO 2000). Conference on, pp. 379-380, 2000.

S. Kobayashi and T. Kimura, "Coherence of Injection Phase-Locked AlGaAs Semiconductor Laser," Electronics Letters, vol. 16, no. 17, pp. 668-670, 141980.

R. Koda, "All-Epitaxial, Long-Wavelength, Vertical-Cavity Surface-Emitting Lasers using Bipolar Cascaded Active Region for High Differential Quantum Efficiency," Ph.D. dissertation, UNIVERSITY OF CALIFORNIA Santa Barbara, 2005.

R. Lang, "Injection Locking Properties of a Semiconductor Laser," IEEE Journal of Quantum Electronics, vol. 18, no. 6, pp. 976-983, Jun 1982.

Y. Lee, J. Jewell, A. Scherer, S. McCall, J. Harbison, and L. Florez, "Room-temperature continuous-wave verticalcavity single-quantum-well microlaser diodes," Electronics Letters, vol. 25, no. 20, pp. 1377-1378, Sept. 1989.

C. Lin and F. Mengel, "Reduction of Frequency Chirping and Dynamic Linewidth in HighSpeed Directly Modulated Semiconductor Lasers by Injection Locking," Electronics Letters, vol. 20, no. 25, pp. 1073-1075, 1984.

N. Margalit, D. Babic, K. Streubel, R. Mirin, R. Naone, J. Bowers, and E. Hu, "Submilliamp long wavelength vertical cavity lasers," Electronics Letters, vol. 32, no. 18, pp. 1675-, Aug 1996.

M. Ortsiefer, M. Lohner, R. Shau, G. Böhm, and M.-C. Amann, "Low-resistance InGaAs tunnel junctions on InP for long-wavelength VCSELs," in Semiconductor and Integrated Optoelectronics Conference (SIOE), Cardiff, 1999.

M. Ortsiefer, R. Shau, G. Bohm, F. Kohler, and M.-C. Amann, "Room-temperature operation of index-guided $1.55 \mu \mathrm{m}$ InP-based vertical-cavity surface-emitting laser," Electronics Letters, vol. 36, no. 5, pp. 437-439, Mar 2000.

M.-R. Park, O.-K. Kwon, W.-S. Han, K.-H. Lee, S.-J. Park, and B.-S. Yoo, “All-epitaxial InAlGaAs-InP VCSELs in the 1.3-1.6- $\mu \mathrm{m}$ Wavelength Range for CWDM Band 
Applications, " IEEE Photonics Technology Letters, vol. 18, no. 16, pp. 1717-1719, Aug. 2006.

J. Piprek, Semiconductor Optoelectronic Devices. Introduction to Physics and Simulation. Academic Press, 2003.

Y. Qian, Z. Zhu, Y. Lo, H. Hou, M. Wang, and W. Lin, "1.3- $\mu$ m Vertical-cavity surfaceemitting lasers with double-bonded GaAs-AlAs Bragg mirrors," IEEE Photonics Technology Letters, vol. 9, no. 1, pp. 8-10, Jan. 1997.

P. Salet, F. Gaborit, P. Pagnod-Rossiaux, A. Plais, E. Derouin, J. Pasquier, and J. Jacquet, "Room temperature pulsed operating of $1.3 \mu \mathrm{m}$ vertical cavity lasers including bottom InGaAsP/InP multilayer bragg mirrors," Electronics Letters, vol. Vol. 33 No. 24, pp. 2048-2049, 1997.

N. Schunk and K. Petermann, "Noise Analysis of Injection-Locked Semiconductor Injection Lasers," IEEE Journal of Quantum Electronics, vol. 22, no. 5, pp. 642-650, May 1986.

R. Shau, M. Ortsiefer, J. Rosskopf, G. Boehm, C. Lauer, M. Maute, and M.-C. Amann, “Longwavelength InPbased VCSELs with buried tunnel junction: properties and applications," SPIE, Vertical-Cavity Surface- Emitting Lasers VIII, vol. 5364, no. 1, pp. 1-15, 2004.

T. Simpson, J. Liu, and A. Gavrielides, "Small-signal analysis of modulation characteristics in a semiconductor laser subject to strong optical injection," IEEE Journal of Quantum Electronics, vol. 32, no. 8, pp. 1456-1468, Aug 1996.

H. Soda; K. Iga, C. Kitahara, and Y. Suematsu, "GaInAsP/InP Surface Emitting Injection Lasers," Japanese Journal of Applied Physics, vol. 18, no. 12, pp. 2329-2330, 1979.

H.-K. Sung, T. Jung, D. Tishinin, K. Liou, W. Tsang, and M. Wu, “Optical Injection- Locked Gain-Lever Distributed Bragg Reflector Lasers With Enhanced RF Performance," IEEE International Topical Meeting on Microwave Photonics. MWP'2004., pp. 225-228, Oct. 2004.

A. Syrbu, V. Iakovlev, G. Suruceanu, A. Caliman, A. Mereuta, A. Mircea, C.-A. Berseth, E. Diechsel, J. Boucart, A. Rudra, and E. Kapon, "VCSELs Emitting in the 1310- nm Waveband for Novel Optical Communication Applications," SPIE Vertical-Cavity Surface-Emitting Lasers IX, vol. 5737, no. 1, pp. 167-173, 2005.

A. Syrbu, V. Iakovlev, G. Suruceanu, A. Caliman, A. Rudra, A. Mircea, A. Mereuta, S. Tadeoni, C.-A. Berseth, M. Achtenhagen, J. Boucart, and E. Kapon, "1.55- $\mu \mathrm{m}$ optically pumped wafer-fused tunable VCSELs with 32-nm tuning range," IEEE Photonics Technology Letters, vol. 16, no. 9, pp. 1991-1993, Sept. 2004.

H. Toba, Y. Kobayashi, K. Yanagimoto, H. Nagai, and M. Nakahara, "Injection-locking Technique Applied to a $170 \mathrm{~km}$ Transmission Experiment at $445.8 \mathrm{Mbit} / \mathrm{s}$," Electronics Letters, vol. 20, no. 9, pp. 370-371, 261984.

K. J. Vahala, M. A. Newkirk, and T. R. Chen, "The Optical Gain Lever: A Novel Gain Mechanism in the Direct Modulation of Quantum Well Semiconductor Lasers," Applied Physics Letters, vol. 54, no. 25, pp. 2506-2508, 1989.

G. Yabre, H. De Waardt, H. van den Boom, and G.-D. Khoe, "Noise Characteristics of SingleMode Semiconductor Lasers Under External Light Injection," IEEE Journal of Quantum Electronics, vol. 36, no. 3, pp. 385-393, Mar 2000.

X. Zhao, M. Moewe, L. Chrostowski, C.-H. Chang, R. Shau, M. Ortsiefer, M.-C. Amann, and C. Chang-Hasnain, "28 GHz Optical Injection-Locked $1.55 \mu \mathrm{m}$ VCSELs," IEE Electronic Letters, vol. Vol. 40 No. 8, 15 April 2004.

X. Zhao, E. K. Lau, D. Parekh, H.-K. Sung, W. Hofmann, M. C. Amann, M. C. Wu, and C. J. Chang-Hasnain, "107- GHz Resonance Frequency of 1.55 $\mu \mathrm{m}$ VCSELs Under Ultrahigh Optical Injection Locking," in OSA/CLEO, 2008. 


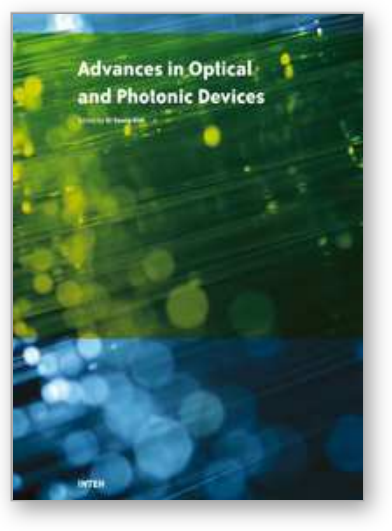

\author{
Advances in Optical and Photonic Devices \\ Edited by Ki Young Kim
}

ISBN 978-953-7619-76-3

Hard cover, 352 pages

Publisher InTech

Published online 01, January, 2010

Published in print edition January, 2010

The title of this book, Advances in Optical and Photonic Devices, encompasses a broad range of theory and applications which are of interest for diverse classes of optical and photonic devices. Unquestionably, recent successful achievements in modern optical communications and multifunctional systems have been accomplished based on composing "building blocks" of a variety of optical and photonic devices. Thus, the grasp of current trends and needs in device technology would be useful for further development of such a range of relative applications. The book is going to be a collection of contemporary researches and developments of various devices and structures in the area of optics and photonics. It is composed of 17 excellent chapters covering fundamental theory, physical operation mechanisms, fabrication and measurement techniques, and application examples. Besides, it contains comprehensive reviews of recent trends and advancements in the field. First six chapters are especially focused on diverse aspects of recent developments of lasers and related technologies, while the later chapters deal with various optical and photonic devices including waveguides, filters, oscillators, isolators, photodiodes, photomultipliers, microcavities, and so on. Although the book is a collected edition of specific technological issues, I strongly believe that the readers can obtain generous and overall ideas and knowledge of the state-of-the-art technologies in optical and photonic devices. Lastly, special words of thanks should go to all the scientists and engineers who have devoted a great deal of time to writing excellent chapters in this book.

\title{
How to reference
}

In order to correctly reference this scholarly work, feel free to copy and paste the following:

Ahmad Hayat, Alexandre Bacou, Angélique Rissons and Jean-Claude Mollier (2010). Optical Injection-Locking of VCSELs, Advances in Optical and Photonic Devices, Ki Young Kim (Ed.), ISBN: 978-953-7619-76-3, InTech, Available from: http://www.intechopen.com/books/advances-in-optical-and-photonic-devices/optical-injectionlocking-of-vcsels

\section{INTECH}

open science | open minds

\section{InTech Europe}

University Campus STeP Ri

Slavka Krautzeka 83/A

51000 Rijeka, Croatia

Phone: +385 (51) 770447

\section{InTech China}

Unit 405, Office Block, Hotel Equatorial Shanghai

No.65, Yan An Road (West), Shanghai, 200040, China

中国上海市延安西路65号上海国际贵都大饭店办公楼 405 单元

Phone: +86-21-62489820 
Fax: +385 (51) 686166

Fax: +86-21-62489821

www.intechopen.com 
(C) 2010 The Author(s). Licensee IntechOpen. This chapter is distributed under the terms of the Creative Commons Attribution-NonCommercialShareAlike-3.0 License, which permits use, distribution and reproduction for non-commercial purposes, provided the original is properly cited and derivative works building on this content are distributed under the same license. 